\title{
3 Funktionen und Kontexte
}

In diesem Kapitel erörtere ich in Kürze die Funktionen und anschließend zur Motivation des weiteren Vorgehens die Kontexte von Füllpartikeln. Abschnitt 3.1 zeigt die Multifunktionalität von Füllpartikeln anhand fünf häufig genannter Funktionen und stellt die Haltung dieser Arbeit zur Signal-Symptom-Debatte dar. Die postulierten Funktionen entstehen fast ausschließlich aus dem linguistischen Kontext von Füllpartikeln. Konsequenterweise müssen Funktionen daher als Interpretation der Kontexte hintan gestellt werden. Abschnitt 3.2 beschreibt folglich die bisher beschriebenen Kontexte und, falls vorhanden, ihre Interaktion mit der phonetischen Realisierung von Füllpartikeln, woraus schließlich die Form-Kontext-Hypothese entwickelt wird. Ferner wird in Abschnitt 3.3 ein Überblick über sprecher- und situationsspezifische Distributionen und Formen von Füllpartikeln gegeben.

\subsection{Multifunktionalität von Füllpartikeln}

\subsubsection{Symptom versus Signal}

Bevor ich die mutmaßlichen Funktionen von Füllpartikeln diskutiere, werde ich zur Einordnung der von dieser Arbeit eingenommenen Perspektive die Debatte um die beiden am häufigsten in der Literatur diskutierten Hypothesen zur Produktion von Füllpartikeln, die Symptomhypothese und die Signalhypothese, in ihren wesentlichen Zügen rekapitulieren. Dies ist notwendig, um die hier vorliegende Arbeit von diesen Hypothesen freizumachen.

Im Rahmen der Symptomhypothese werden Füllpartikeln als Epiphänomene der Sprachproduktion betrachtet und häufig als Häsitation oder gefüllte Pause bezeichnet. Ursächlich für ihre Verwendung seien Planungsansprüche an die Sprachproduktion (Beattie \& Butterworth 1979; Beattie \& Shovelton 2002; Blackmer \& Mitton 1991; Corley \& Stewart 2008; Finlayson \& Corley 2012; Lay \& Paivio 1969; Levelt 
1983, 1989; Maclay \& Osgood 1959; Martin \& Strange 1968; Oviatt 1995; Rochester 1973). Neben dieser psycholinguistischen Erklärung haben Schönle \& B. Conrad (1985) versucht, eine physiologische Erklärung zu geben. Sie untersuchen 16 deutsche Muttersprachler (im Mittel 26,4 Jahre alt), die fünf Minuten Spontansprache produzieren und deren Atmung mithilfe eines Atmungsmessgürtels aufgezeichnet wird, um zu ermitteln, an welchen Stellen ihrer Ausatmungsphase Füllpartikeln auftreten, wobei die Ausatmungsphase in vier äquidistante Bereiche aufgeteilt wird. ${ }^{54}$ 62,5\% aller Füllpartikelvokale treten im ersten Bereich auf, 20,8 \% im zweiten, 2,1 \% im dritten und 14,6\% im vierten (ebd.: 294f.). Aus diesen Ergebnissen schließen Schönle \& B. Conrad, dass „hesitation vowels" als phonomotorische Subroutinen des Sprachproduktionssystems verwendet werden, um für fehlendes Sprachmaterial zu kompensieren und das respiratorische System vor unökonomischem Luftverlust zu bewahren. Obwohl beide Erklärungen, die psycholinguistische und die physiologische, Ähnlichkeiten hinsichtlich ihres symptomatischen Charakters aufweisen, sind sie bisher nicht zusammen betrachtet worden.

Im Rahmen der Signalhypothese wird argumentiert, dass Füllpartikeln ein intentionales Signal an einen Hörer oder eine Hörerin darstellen (Clark \& Fox Tree 2002; Eklund 2004; Goodwin 1981; Jefferson 1974; Smith \& Clark 1993; E. J. Walker et al. 2014; Willkop 1988). Beispiel für ein solches Signal sei die Verwendung (im Englischen) für „comments on the on-going performance“ (Clark \& Fox Tree 2002: 107) oder die Verwendung als Turnhalte- oder Turnabgabesignal („I want to keep the floor" und "I want to give up the floor"), die Verwendung als Unsicherheitssignal („I'm uncertain about what I want to say“) oder zur Anzeige von Wortfindungsanstrengungen („I'm hunting for the next word“) (ebd.: 104). In Abschnitt 3.1.2 werden diese Funktionen eingehender betrachtet. Hier scheint schon die Schwierigkeit einer klaren phänomenalen und epistemologischen Trennung durch; der gleiche Sachverhalt wird mit subtilen terminologischen Unterschieden von der signalhypothetischen Seite als „hunting for the next word“, von der symptomhypothetischen Seite hingegen als Häsitation analysiert. Auf der einen Seite steht die aktive, signalisierende Interpretation des Phänomens im Vordergrund, auf der anderen Seite die passive, durch Schwierigkeiten in der Sprachplanung entstandene Verzögerung.

Ein vorgeschlagener Kompromiss für das Dilemma von Symptom und Signal be-

\footnotetext{
${ }^{54}$ Fünf der Probanden verwenden keine Füllpartikeln (Schönle \& B. Conrad 1985: 294).
} 
steht darin, Füllpartikeln als Reflex der Sprachplanung zu betrachten, die dann (quasi indexikalisch) ${ }^{55}$ von der Hörerseite verarbeitet werden (Arnold et al. 2003, 2004; Brennan \& Williams 1995; Corley et al. 2007; Ferreira \& Bailey 2004; Finlayson \& Corley 2012; Watanabe et al. 2008). Für das Deutsche hat Keseling (1989) versucht, die Symptom- und die Signalperspektive zu vereinen.

Wenn es zutrifft, dass ÄH vor einer wichtigen Aussage oder einem wichtigen Wort plaziert ist und wenn es zugleich an Stellen auftritt, die vom Sprecher (verstärktes) Nachdenken erfordern, dann scheint die Hauptfunktion dieses Lautgebildes in der Koordination von planerischem Nachdenken auf der Sprecherseite und (erhöhter) Aufmerksamkeit auf der Hörerseite zu bestehen (ebd.: 589).

In diesem Sinne schreibt auch Reitbrecht (2017) Füllpartikeln „Sprachverarbeitung stützende Funktionen" zu und folgert, dass der Ansatz, Füllpartikeln jeweils apodiktisch mittels einer einzigen dieser beiden funktionalen Hypothesen erklären zu wollen, scheitern muss. Reitbrecht nimmt die „sprechplanerische, kognitive Funktion“ als „potenziell gegeben [Im Original kursiv - M. B.]" an, und versteht die Symptom- und die Signalhypothese als „potenziell simultan verlaufende Komplementärfunktionen [Im Original kursiv - M. B.] [. . .], die einander nicht ausschließen“ (ebd.: 27).

Ich schließe mich hier der Sicht von Reitbrecht an, da der Beitrag dieser Arbeit nicht die Lösung der Symptom-Signal-Debatte anstrebt. Vielmehr möchte ich eine gebrauchsbasierte Erklärung der verwendeten phonetischen Formen von Füllpartikeln in ihren linguistischen Produktionskontexten geben. Ich nehme an, dass FormKontext-Muster existieren (vgl. Abschnitt 3.2.1), die dann in zukünftigen Arbeiten zu den oben beschriebenen Erklärungsansätzen weiter diskutiert werden können. Bevor diese Hypothese jedoch genauer ausgeführt wird, beschreibe ich im nächsten Abschnitt einige Funktionen, die Füllpartikeln zugeschrieben werden. Die Unauflösbarkeit der verschiedenen Top-Down-Funktionszuschreibungen und ihre Rückführung auf die Auftretenskontexte motiviert die weitere Betrachtungsweise im Verlaufe dieser Arbeit.

\footnotetext{
${ }^{55}$ Fischer (1999) votiert sogar für einen ikonischen Charakter von Füllpartikeln.
} 


\subsubsection{Funktionen}

Ohne also weiter auf die Symptom-Signal-Debatte einzugehen, bespreche ich hier in gebotener Kürze einige häufig identifizierte sprachliche Funktionen von Füllpartikeln. Im linguistischen Sinne ist der Begriff ,Funktion‘ polysem und kann je nach linguistischer Disziplin variieren (beispielsweise „Intonation [als] satztypendifferenzierende F[unktion]" oder kommunikative Aspekte in der linguistischen Pragmatik, vgl. Glück 2005: 206). Hier verstehe und verwende ich den Begriff der Funktion zunächst bewusst grob als ,interne oder externe Beziehung zwischen ling[uistischen] Sachverhalten" (ebd.).

Kjellmer (2003) votiert für die Multifunktionalität von Füllpartikeln im Englischen und gegen eine rein häsitatorische Funktion, denn „[h]esitation may not always be involved" (ebd.: 171). Als Argument für die Multifunktionalität gibt Kjellmer Beispiele, dass Füllpartikeln thought units (,Denkeinheiten') auf Wort-, Phrasenund Satzebene einführen (ebd.: 174-181), Redewechsel steuern (ebd.: 186), die Salienz nachfolgender Wörter erhöhen (ebd.: 187), als Korrektureinheiten dienen (ebd.: 188f.), und als strukturelle Einheiten verwendet werden können (ebd.: 190).

Im Kern sind dies also fünf Funktionen, welche auch in der englischen und deutschen Literatur (vgl. unten) immer wieder genannt werden, nämlich die Verwendung als Verzögerungs- beziehungsweise Häsitationsmarker (f.hes), die Verwendung als redewechselbezogener Marker beziehungsweise Turnmarker (f.turn), die Verwendung als Reparaturmarker (f.rep), die Verwendung als prominenz- beziehungsweise salienzbezogener Marker (f.sal) und die Verwendung als struktureller beziehungsweise einführender Marker, was sich in den Grenzen der Dialogbetrachtung (vgl. Abschnitt 3.2.3.3) als dialogstrukturelle Verwendung (f.dia) auffassen lässt. Diese Funktionen stehen in keiner hierarchischen Abhängigkeit zueinander. Während die Funktionen f.turn und f.dia auf einer dialogischen Struktur aufbauen, können f.hes, f.sal und f.rep sowohl in Dialogen als auch in Monologen auftreten.

Im Folgenden wird jede dieser Funktionen aus Erwähnungen in der Literatur zum Englischen und Deutschen (sofern vorhanden) heraus näher beschrieben, bevor nochmals zwei deutschsprachige Studien skizziert werden, welche die fünf Funktionen in ähnlicher Weise fürs Deutsche postulieren. Generell ist fraglich, ob die Ergebnisse der Füllpartikelstudien im Englischen auf die Verwendung von Füllpartikeln im Deutschen eins zu eins übertragen werden können. Des weiteren ist die Ermittlung 
einer Funktion keineswegs eindeutig, sondern kann in hohem Maße mit anderen Funktionen überlappen. Dies ist einerseits der Vielfalt der verwendeten Methoden geschuldet, die von psycholinguistischen und konversationsanalytischen bis hin zu korpuslinguistischen Ansätzen reichen, andererseits fehlt auch eine Entscheidungshilfe. Obwohl die funktionale Analyse in vielen Fällen durch ihren Kontext geleitet werden kann, bleibt sie hochsubjektiv.

Funktion f.hes - Verzögerungsmarker: Die Verwendung von Füllpartikeln als Häsitations- oder Verzögerungsmarker (f.hes) lässt sich größtenteils aus den Untersuchungen zu Füllpartikeln als Symptom ableiten (Blackmer \& Mitton 1991; Clark \& Fox Tree 2002; Corley \& Stewart 2008; Finlayson \& Corley 2012; Goldman-Eisler 1961; Lay \& Paivio 1969; Levelt 1989; Lickley 2015; Martin \& Strange 1968; Rochester 1973). Füllpartikeln in dieser Funktion werden demnach produziert, wenn die Belastung durch die Sprachplanung zu groß wird („when speech is interrupted at or close to trouble“, Levelt 1983: 74). Dies kann beispielsweise durch den erhöhten Schwierigkeitsgrad einer zu lösenden Aufgabe induziert werden (vgl. Rochester 1973: 70). So produzierten 24 männliche englischsprachige Versuchspersonen Füllpartikeln bei Selbstbeschreibungen in 2,8\% aller Wörter, bei Cartoonbeschreibungen in 5,7\% und bei der Erklärung von Sprichwörtern in $9 \%$ aller Wörter (Lay \& Paivio 1969: 31). f.hes ist quasi der Prototyp für die funktionale Verwendung von Füllpartikeln. Die Funktion f.hes basiert auf Annahmen über die Sprachplanung eines Sprechers oder einer Sprecherin. Es lässt sich in der Literatur keine Präferenz für eine spezifische Position innerhalb von linguistischen Kontexten feststellen.

Funktion f.rep - Reparaturmarker: Diese Funktion beschreibt die Verwendung von Füllpartikeln in Verbindung mit Reparaturen oder Neustarts (Belz 2013; Belz et al. 2017; Heeman \& Allen 1999; Levelt 1983; Seyfeddinipur et al. 2008; Shriberg 1994). Auf den ersten Blick ist die Funktion einer Füllpartikelentität relativ einfach zuweisbar, steht sie doch innerhalb einer Reparatur, deren Struktur bekannt ist (vgl. bspw. Shriberg 1994). Prinzipiell wäre jedoch auch die Zuschreibung von f.hes zu solch einer Füllpartikel innerhalb einer Reparatur denkbar - dass Füllpartikeln innerhalb von Reparaturen die Funktion f.rep erhalten, ist also vor allem durch den gut definierbaren Kontext und das Auftreten in eben diesem bedingt. Während - vielleicht auch aufgrund dieser nicht eineindeutigen Zuweisbarkeit - über die Häufigkeit von f.hes bisher keine Aussagen getroffen worden sind (es fehlt 
eine funktionsbasierte Korpusstudie), kann die Häufigkeit von f.rep gezielt ermittelt werden. Beispielsweise finden sich in BeMaTaC ${ }^{56}$ (Sauer \& Lüdeling 2016), einem Map-Task-Korpus mit 12 aufgabenbasierten Dialogen 16 deutscher Muttersprachlerinnen und Muttersprachler (11 199 Token), insgesamt 210 Reparaturen $^{57}(=1,9 \%$ aller Token), in denen wiederum 29 Füllpartikeln ${ }^{58}$ enthalten sind. Bei insgesamt 274 Füllpartikeln ${ }^{59}$ (äh und $\ddot{a} h m$ ) beträgt demnach der Anteil von Füllpartikeln mit der Funktion f.rep in diesem Korpus 10,6\%.

Funktion f.sal - Salienz und Informationsstatus: Diese Funktion beschreibt die Verwendung von Füllpartikeln als Signal für etwas Neues, zur Hervorhebung und zur Herstellung von Aufmerksamkeit. Der Status von Füllpartikeln im Zusammenhang mit Informationsstruktur ${ }^{60}$ ist bislang noch umstritten, wenn auch die Hypothese, dass Disfluencies (und in Folge Füllpartikeln) im Allgemeinen eher vor nicht-vorerwähnten Referenten vorkommen, schon lange im Raum steht (Maclay \& Osgood 1959: 34). Auch wurde in psycholinguistischen Eye-Tracking-Studien für die englische Sprache festgestellt, dass Füllpartikeln häufiger vor neuer Information als vor gegebener Information produziert und erwartet werden (Arnold et al. 2003; Arnold \& Tanenhaus 2011; Arnold et al. 2004). Für das Deutsche lässt sich mithilfe des eben schon herangezogenen Korpus BeMaTaC zeigen, dass etwa die Hälfte, nämlich 15 der 29 Füllpartikeln in Reparaturen, vor einem „neuen“, im linken Teil der Reparatur nicht vorerwähnten Token stehen. ${ }^{61}$ Faure (1980: 290) hingegen findet keinen Zusammenhang zwischen Thema-Rhema-Struktur und Füllpartikeln in französischen und deutschen Narrativen, Diskussionen und spontansprachlichen

\footnotetext{
${ }^{56}$ Berlin Map Task Corpus v. 3.0, http://u.hu-berlin.de/bematac, besucht am 20.05.2019.

${ }^{57}$ Da Reparaturen symmetrisch aus Reparandum und Reparans bestehen, werden für diesen Zweck nur die Reparaturanfänge (Reparanda) herangezogen, vgl. https://korpling.org/annis3/ ?id=bb9aaf91-630c-490f $-9 e c d-3 f 82557 e c 7 b 6$, besucht am 24.04.2019.

${ }^{58} \mathrm{https}$ ://korpling.org/annis3/?id=4e88a086-da66-42e3-ad72-7f844bf $2 \mathrm{ceec}$, besucht am 24.04.2019.

${ }^{59}$ https : //korpling.org/annis3/?id=56f9b849-fd67-40aa-8519-bd4090516b22, besucht am 24.04.2019.

${ }^{60}$ Informationsstruktur untersucht ,die Art, wie Sätze an den Kontext angepasst werden hinsichtlich Dimensionen wie Bekanntheit/Unbekanntheit, Topik/Kommentar und Fokus/Hintergrund" (Musan 2010: 3; vgl. auch Krifka \& Musan 2012). Diese Dimensionen beziehen sich dabei auf verschiedentlich große Konstituenten und werden besonders im Kontext geschriebener Sprache angewendet.

${ }^{61}$ Da der rechte Teil einer Reparatur auch aus mehreren Token bestehen kann, wird aufgrund der grammatischen Struktur des Deutschen das letzte Token in der Abfolge auf seine Vorerwähntheit hin evaluiert, https://korpling.org/annis3/?id=9c932fe4-551c-4e2e-84a5-716c1166af0d, besucht am 20.05.2019.
} 
Konversationen.

Ein weiterer Aspekt von f.sal ist das Herstellen von Aufmerksamkeit, wie es (anekdotisch) beim Ansprechen eines Menschen verwendet werden kann, beispielsweise in $\ddot{a} h$ Entschuldigung. Tatsächlich treten hier keine starken Belastungen durch die Sprachplanung auf, wie sie für f.hes postuliert werden. Dennoch überschneidet sich die Funktion mit f.turn und f.dia (s. unten). Ein ähnliches Beispiel für Aufmerksamkeitserheischung gibt Lickley (2015) für das Englische mit „, um, excuse me! " (ebd.: 463) und erwähnt auch den steigenden Tonhöhenakzent auf um, der als Intention gemeint sein kann, dass ein Sprecher etwas sagen möchte. Das ist mit einiger Wahrscheinlichkeit aber nicht allein das Verdienst des um mit steigendem Ton, sondern sicherlich auch induziert von dem Fragekontext, der oft mit steigendem Ton belegt ist.

Funktion f.turn - Turn-Marker: In der Diskussion ihrer Studie von 163 Äußerungen 13 englischsprachiger männlicher Sprecher auf einer wissenschaftlichen Konferenz schlagen Maclay \& Osgood (1959: 41) vor, dass der Hauptunterschied zwischen stiller und gefüllter Pause in der Dauer des Intervalls liegt, in dem nicht gesprochen wird. Sie nehmen an, dass der momentane Sprecher solange den „conversation ,ball““ behalten möchte, „until he has achieved some sense of completion“ (ebd.). Da ungefüllte Intervalle einer gewissen Länge zum Verlust dieses Rederechts führen könnten, verwendet der Sprecher neben anderen Strategien eine Füllpartikel (ebd.). Seit dieser Studie wird Füllpartikeln eine turnmarkierende Funktion zugeschrieben. Der Begriff Turn wird in der Konversationsanalyse verwendet und beschreibt einen Redebeitrag (Levinson 2000: 323) im Kontext des Sprecherwechsels (Sacks et al. 1974; Schegloff et al. 1977), wobei jedoch in den von mir rezipierten Arbeiten keine genauere Definition über die Ausdehnung des Begriff zu finden war. Dass 56\% aller Füllpartikeln im London-Lund-Corpus (Svartvik 1990) am Anfang einer Intonationsphrase stehen, interpretieren Clark \& Fox Tree als turnmarkierende Verwendung von Füllpartikeln (Clark \& Fox Tree 2002: 94). Kritischerweise ist hinzuzufügen, dass zwar jeder neue Turn mit dem Beginn einer Intonationsphrase zusammenfällt, der Beginn eine Intonationsphrase jedoch nicht immer einen neuen Turn markiert.

Lallgee \& Cook (1969) finden keine Indizien für die von Maclay \& Osgood (1959) postulierte Turn-holding-Funktion von Füllpartikeln, da sie in Interviews mit 14 englischen Muttersprachlern mit Interviewern, die wenig oder viel Druck auf das 
Rederecht des Interviewten erzeugten, keine signifikanten Unterschiede in der Füllpartikelanzahl finden (Lallgee \& Cook 1969: 27). Aufgrund des hohen Drucks auf das Rederecht wurde erwartet, dass die Interviewten häufiger Füllpartikeln nutzen, um den Turn zu halten. Möglicherweise nutzen sie aber andere Strategien. In einer weiteren Studie elizitieren Lalljee \& Cook (1974) Füllpartikeln von 30 männlichen Universitätsstudenten des Englischen unter drei Bedingungen. In der ersten Bedingung monologisiert der Proband zu Themen, die er vor sich auf Karten notiert findet. In der zweiten Bedingung unterhält er sich mit einem Interviewer über die gleichen Themen in der gleichen Reihenfolge. Schließlich diskutiert der Proband die Themen nochmals mit dem Interviewer in einer Art freiem Gespräch. Die Anzahl der produzierten Füllpartikeln je Bedingung unterscheidet sich nicht signifikant (Mono$\log 1,81 \%$, Interview 3,67\%, Konversation 2,83\%), woraus die Autoren schließen, dass in diesem Experiment keine Evidenz für die Turn-holding-Hypothese gefunden wird. Allerdings wird vorausgesetzt, dass die Verwendung im Sinne von f.turn sich auf die Produktion von Füllpartikeln überdurchschnittlich stark auswirkt, da Füllpartikeln auch mit anderen Funktionen verwendet werden können. Mangels einer detaillierten Analyse ist somit nicht klar, was die Häufigkeitsergebnisse aussagen. Lalljee \& Cook (ebd.) schlagen als Alternativerklärung zur Turn-holding-Funktion f.turn Aufmerksamkeitserheischung (,attention-getting') vor, damit der frühe Teil der zu übermittelnden Message nicht verloren geht (ebd.: 224). Dies entspräche der Funktion f.sal. Tatsächlich wird die Multifunktionalität von Füllpartikeln und ihre mögliche Auswirkung auf die phonetische Realisierung bei Lalljee \& Cook (ebd.) schon angedacht:

Perhaps filled pauses performing different functions are different in certain other respects. Some means could be sought to distinguish between various kinds of filled pauses, and grammatical location and extra-linguistic factors such as pitch or volume may be relevant criteria. (ebd.: 224)

Auch in einer rezenteren Studie im Englischen wird kein Indiz für die Verwendung von Füllpartikeln im Sinne von f.turn erbracht. Tottie (2015) analysiert das erste Viertel des Santa Barbara Corpus of American English (SBC1) mit 62330 Wörtern aus 14 verschiedenen Konversationen von 20-25 Minuten Länge. Zwar kommen Füllpartikeln im SBC1 am häufigsten in turn-medialer Position vor $(73 \%)$, jedoch gibt es kaum Fälle in diesen Instanzen, in denen andere Teilnehmer den Turn ergrei- 
fen möchten (sofern sie dies akustisch angezeigt haben und dies auch transkribiert wurde). Somit dienen die Füllpartikeln in turn-medialer Position eher der Planung des verbleibenden Turns (vgl. Tottie 2015: 402-403). Am zweithäufigsten stehen Füllpartikeln in turn-initialer Position (15\%), am dritthäufigsten in turn-finaler Position (6\%) und in turn-zweiter Position (6\%) (ebd.: 393). Sie schlägt als ergänzende Funktion vor, dass man in turn-finalen Positionen signalisiert, nicht weitersprechen zu wollen („diminished intent to continue“) (ebd.: 392). $38 \%$ der Füllpartikeln in turn-initialer Position kommen in Antworten vor (ebd.: 394), was nahelegt, die Dialogstruktur als erklärende Variable genauer zu betrachten. Zusammenfassend wird in der Füllpartikelforschung zwar immer wieder auf die Funktion f.turn rekurriert, ihr Status ist aber weniger gut belegt als die Literatur suggeriert.

Funktion von f.dia - Dialogstruktur: Neben der Verwendung von Füllpartikeln zu Beginn, am Ende oder innerhalb von dialogstrukturierenden Einheiten (Dialogzüge, vgl. Abschnitt 3.2.3.3) treten Füllpartikeln im Deutschen auch „unmittelbar nach der Begrüßung oder nach der Selbstidentifikation auf, vor dem Einstieg in das Thema“ (Rasoloson 1994: 61f.). Dies deckt sich mit der Beobachtung, dass ein uhm zu Beginn eines Telefonanrufes als strukturierendes Mittel steht, „which gives no indication here of uncertainty, reluctance, hesitation, trouble, or the like" (Schegloff 2010: 147f.). Somit gibt es eine Schnittmenge zwischen f.dia und f.sal. In der Funktion f.dia müssen Füllpartikeln jedoch nicht zwangsläufig Aufmerksamkeit erregen, da in in Frage-Antwort-Paarsequenzen die Aufmerksamkeit ja schon hergestellt wurde - analog muss hier auch kein Turn beansprucht oder gehalten werden (f.turn). Dennoch stehen Füllpartikeln in englischen aufgabenbasierten Dialogen häufiger vor Antworten als vor anderen dialogstrukturellen Einheiten (Lickley 2001). Für das amerikanische Englisch liegt in ähnlichen Dialogen keine ausreichende Bestätigung vor (Nicholson et al. 2010). Auf beide Studien gehe ich in Abschnitt 3.2.3.3 näher ein. Im Deutschen dienen Füllpartikeln laut Schwitalla (2002) „dem prospektiven Sprecher zur Redeplanung und signalisieren, dass er/sie bereit ist, Antwortobligation zu übernehmen“ (ebd.: 274). Die funktionale Beschreibung von Füllpartikeln als „attention-getting signal“ (Lalljee \& Cook 1974: 224), was vor dem neuen Sprecheinsatz stehen $\mathrm{kann}^{62}$, kann sowohl dialogstrukturierend als f.dia wie auch als f.sal oder als f.turn interpretiert werden.

\footnotetext{
${ }^{62}$ "A person may say ,er' before he begins speaking in order to draw attention to himself either to signal that he would like to speak or that he is about to speak." (Lalljee \& Cook 1974: 224).
} 
Nachdem die oben aufgeführten fünf Funktionen vornehmlich englischsprachige Studien wiedergeben, seien kurz die zwei deutschen konversationsanalytischen Studien erwähnt, die maßgeblich mit einer eigenen Analyse zu ähnlichen Schlussfolgerungen gekommen sind. Willkop (1988) untersucht Füllpartikeln vornehmlich in Alltagsgesprächen ${ }^{63}$ und schreibt Füllpartikeln eine „primär redeorganisierende Funktion" zu; sie können in diesem Sinne Unterbrechungen seitens des Gegenübers verhindern, Formulierungsschwierigkeiten überbrücken, eine Turn-Übernahme anmelden und seien ein „Signal für eine nicht fremdinitiierte Eigenkorrektur“ (ebd.: 251). Rasoloson (1994) untersucht Füllpartikeln in 132 deutschen „Informationsdiskursen" (Alltagssituationen, in welchen eine Information eingeholt wird) zwischen 1975 und 1986 (ebd.: 28 f.). Rasoloson (ebd.: 79) analysiert Füllpartikeln als Steuerungsmittel (Bearbeitung des Turn-/Musterabbruchs, Anspruch auf Sprecherrolle), als Korrekturausdruck, als Gliederungsmittel (Ankündigung eines neuen Inhaltsabschnitts, Markierung der Verteilung der Sprecher- und Hörerrolle), als deliberatives Ausdrucksmittel $^{64}$ (zur Lexemsuche und (Teil)Satzbearbeitung, vor Informationsfrage und vor positiver Antwort) und als Indikator für einen Erwartungsbruch (vor negativer Antwort).

In Tabelle 3.1 fasse ich die oben zusammengefassten meistbeschriebenen ${ }^{65}$ Funktionen verkürzt unter ihren Abkürzungen zusammen. Die fünf dort beschriebenen Funktionen sind jedoch nicht immer disjunkt zueinander, im Gegenteil, sie haben große Schnittmengen. Des weiteren werden sie methodisch unterschiedlich übermittelt. Indikatoren für die Feststellung einer Funktion sind hauptsächlich die linguistischen Kontexte, in denen sie auftreten. So kann eine Füllpartikel vor einer Frage auch als Füllpartikel vor Turn-Beginn klassifiziert werden (f.turn und f.dia), wenn an dieser Stelle ein Turn beginnt. Zusammenfassend sind die Funktionen von Füllpartikeln im Sinne der von Fedriani \& Sansò (2017) entwickelten Terminologie zur Beschreibung von diskursmarkierenden Einheiten prozedural (f.hes), indexikal (f.turn, f.sal, f.dia) und metalinguistisch (f.rep). Die Funktionen f.turn und f.dia beschreiben

\footnotetext{
${ }^{63}$ Mindestens zwei Alltagsgespräche, ergänzt mit Telefondialogen und verschiedenen weiteren Daten. Der Gesamtumfang der Daten war nicht auszumachen (vgl. Willkop 1988: 14 f.).

${ }^{64}$ Zum Verwendung des Terminus deliberativ im Sinne von überlegt, bedacht, erwogen in diesem Kontext vgl. „a deliberate signal chosen from a range of interjections" (Smith \& Clark 1993: 27) und „als deliberatives Ausdrucksmittel“ (Rasoloson 1994: 77).

${ }^{65}$ Weitere Funktionen sind nicht ausgeschlossen. Weniger häufig erwähnt wird beispielsweise die Verwendung von $u h$ im Englischen als pragmatischer Markierer zur Zurückweisung einer Implikatur (Norrick 2009: 875).
} 
Funktionen, die besonders in interaktionalen Situationen auftreten. Die Funktionen f.hes, f.rep und f.sal können sowohl in monologischen als auch dialogischen Situationen auftreten.

Tabelle 3.1: Nichtexhaustive Zusammenstellung und Beschreibung der meistangenommenen Funktionen von Füllpartikeln in bestimmten Positionen, wie sie in der Literatur postuliert werden. Die Funktionen sind nicht hierarchisch organisiert.

\begin{tabular}{|c|c|c|}
\hline Abkürzung & Funktion & Position \\
\hline f.hes & Markiert Verzögerung & $\begin{array}{l}\text { Häufige Interpretation einer Füllpartikel, wenn kei- } \\
\text { ne spezifischere Funktion zutrifft. }\end{array}$ \\
\hline f.rep & Markiert Reparaturen & Steht innerhalb von Reparaturen. \\
\hline f.turn & Markiert Turns & $\begin{array}{l}\text { Steht am Anfang (Redeübernahme), in der Mit- } \\
\text { te (Erhalt des Redebeitrags) oder am Ende eines } \\
\text { Turns (Abgabe oder Erhalt des Redebeitrags). }\end{array}$ \\
\hline f.sal & $\begin{array}{l}\text { Markiert Aufmerksamkeit, } \\
\text { Salienz oder Informations- } \\
\text { status }\end{array}$ & $\begin{array}{l}\text { Steht zu Beginn der Gesprächseröffnung, vor neuen } \\
\text { Inhaltsabschnitten, vor neuen Referenten. }\end{array}$ \\
\hline f.dia & Markiert Dialogstruktur & $\begin{array}{l}\text { Steht vor Fragen, vor Antworten, innerhalb von } \\
\text { Narrativen. }\end{array}$ \\
\hline
\end{tabular}

Dieses Kapitel und Tabelle 3.1 zeigen, dass eine Funktionsbestimmung von Füllpartikeln nicht losgelöst von den sie umgebenden Kontexten durchgeführt werden kann. Tatsächlich ergeben sich die herausgearbeiteten Funktionen in den meisten Fällen gerade erst durch die Berücksichtigung ihres Kontextes, oder anders formuliert, der Kontext einer Füllpartikel stellt das definitorische Kriterium für ihre funktionale Interpretation. Die Funktion f.rep wird durch den Reparaturkontext, f.turn durch den Turnkontext, f.dia durch den diskursstrukturellen Kontext und f.sal durch den informationsstrukturellen Kontext definiert. Falls sich keine der spezifischeren Funktionen ableiten lässt, bleibt immer noch die Funktion f.hes. Eine spezifische Ausprägung einer Füllpartikel kann dabei auch mehrere Funktionen erfüllen. Nachdem beispielsweise ein Turn von Gesprächspartner A mit einer Frage beendet wurde, kann eine Füllpartikel vor der Antwort des Gesprächspartners B sowohl als Turnfunktion (f.turn) als auch als dialogstrukturierende Funktion (f.dia) interpretiert werden - mangels klarer ,Beweise‘ muss sie es sogar; sie könnte aber natürlich auch die Funktion f.hes erhalten. Wenn - als zweites Beispiel - eine Füllpartikel innerhalb eines ansonsten flüssigen Redebeitrags vorkommt, so wird ihr oftmals (mangels anderer Indizien) die Verwendung als f.hes zugeschrieben. Findet sich die Füllpartikel allerdings innerhalb einer Reparatur, so erhält sie in dem 
vorherrschenden top-down-funktionsbasierten Ansatz die Funktion f.rep. Dies findet schon Shriberg (1994) problematisch:

An alternative solution (which represents the implicit view in some past accounts) proposes that filled pauses that occur in the IM [interregnum - M.B.] of a longer DF [disfluency - M.B.] function as editing phrases, whereas those occurring elsewhere function as hesitations. There is to date no hard evidence for this view. (ebd.: 64)

Zusammenfassend wurde gezeigt, dass sich Funktionszuschreibungen für Füllpartikeln nolens volens an dem Kontext der Füllpartikel orientieren und dass die Festlegung einer bestimmten Funktion hochgradig interpretativ, methodisch divers und subjektiv ist. Ich werde daher davon Abstand nehmen, Funktionen für Füllpartikeln top down oder auf andere Weise zuzuweisen. Im folgenden Abschnitt stelle ich die Form-Kontext-Hypothese vor und diskutiere, wie sich dem Desiderat der fehlenden „hard evidence“ (ebd.) für Funktionszuschreibungen über den Umweg der kontextbasierten Analyse angenähert werden kann.

\subsection{Kontext und phonetische Realisierung}

In diesem Abschnitt stelle ich die Form-Kontext-Hypothese auf und erläutere sie mit Beispielen und einem Literaturüberblick.

\subsubsection{Die Form-Kontext-Hypothese}

Only when we look at the way speakers employ different phonetic means to organize the most common form of spontaneous speech, conversation, do patterns occur which are exclusive to spontaneous speech. (Simpson 2013: 169)

Simpson betont die immanente phonetische Relevanz für die Beschreibung des Forschungsgegenstands der spontanen gesprochenen Sprache und ihrer verwendeten Gebrauchsmuster. Ohne eine phonetische Beschreibungsebene bleibt die Beschreibung gesprochener Sprache und der in ihr von Sprecher*innen verwendeten Phänomene defektiv. Gerade für die Betrachtung genuin gesprochensprachlicher Phänomene, wie sie Füllpartikeln darstellen, können mithilfe phonetischer Merkmale bisher unbekannte Verwendungsmuster beschrieben, erklärt und eingeordnet werden. 
Mit der Form-Kontext-Hypothese bezeichne ich die Vermutung, dass es eine systematische Beziehung zwischen phonetischer Realisierungsform und linguistischem Kontext dergestalt gibt, dass die Variabilität der verwendeten phonetischen Füllpartikelformen mit ihren linguistischen Auftretenskontexten korreliert. Mit Kontexten bezeichne ich die linguistischen Auftretenskontexte von Füllpartikeln - diese können sowohl sequenzieller als auch hierarchischer Art sein, also beispielsweise die die Füllpartikel umgebenden, adjazenten Lautsegmente (sequenziell) oder das Vorkommen in einer Antwort (hierarchisch). Eine Füllpartikel nimmt in ihrem Kontext dann eine bestimmte Position ein - sequenziell zwischen Segmenten, Lauten oder Wörtern, oder hierarchisch an den Grenzen oder innerhalb von Phrasen oder höheren Einheiten.

Wie in Abschnitt 3.1.2 erörtert, dient in den meisten Fällen der Auftretenskontext beziehungsweise die Position einer Füllpartikel als Indiz für ihre unterstellte Funktion. Weitere Evidenzen für diese Annahme fehlen bisher jedoch. Fraglich bleibt zudem, inwiefern Füllpartikeln in den verschiedenen Kontexten dann tatsächlich die ihnen zugeschriebenen Funktionen übernehmen. Es bleibt offen, ob dies überhaupt mit Sicherheit beantwortet werden kann. Daher besteht hier ein Desiderat der Beschreibung kontextueller Verwendungen von Füllpartikeln, welches ich zudem mit dem Desiderat der Beschreibung phonetischer Realisierungen verknüpfe. Wenn Füllpartikeln verschiedene phonetische Formen zeigen (wofür es starke Indizien gibt), kann überprüft werden, ob bestimmte Formen charakteristisch für bestimmte Kontexte sind und sie dort über- oder mindergebraucht werden. Meine Hypothese ist, dass sich aufgrund der hohen Formvariabilität von Füllpartikeln (vgl. Kapitel 2) möglicherweise phonetische Korrelate bestimmter Auftretenskontexte finden lassen, die die These stützen, dass es kontextuell unterschiedliche Verwendungsmuster und damit unterschiedliche Form-Kontext-Paare gibt. Mithilfe dieses innovativen Ansatzes wird zunächst getrennt voneinander die Form und der Kontext von Füllpartikeln beschrieben. Dies bedeutet, dass Kontexte nicht nur in der Umgebung einer Füllpartikel beschrieben werden, sondern sämtliche in den Daten enthaltenen ausgesuchten Kontexte, sodass eine nachfolgende Analyse auch Aussagen darüber treffen kann, in welchen Kontexten und wie häufig keine Füllpartikel vorkommt. Durch die Vermeidung einer holistischen Top-down-Funktionszuweisung und die Wahl des umgekehrten Weges, eines auf Oberflächenmerkmalen basierenden Bottom-up-Ansatzes, 
wird die Analyse von Form und Kontext separat nachprüfbar und replizierbar. Dies ist insofern eine adäquate Herangehensweise an die Beschreibung dieses Phänomens, als eine eineindeutige Funktionszuordnung nicht möglich ist. Die produzierten Formen können so jedoch unabhängig von ihren Kontexten beschrieben und systematisiert werden. Erst in der anschließenden korpusbasierten Analyse wird dann eine kombinierte Auswertung von phonetischer Form und linguistischem Kontext vorgenommen. Die Analyse des Audiosignals in den Kontexten ist ein zusätzliches, jedoch unerlässliches Novum und ermöglicht eine detaillierte Beschreibung der Füllpartikeln in Bezug auf die phonetisch-akustischen Korrelate ihrer Verwendung.

Darüber hinaus könnte man evaluieren, inwiefern die angenommenen Funktionen aus Abschnitt 3.1.2 mit den emergierten Form-Kontext-Mustern korrelieren. Die hier vorgeschlagene Analyse lässt auch die Analyse von Kontextdistributionen zu, in denen keine Füllpartikel auftritt, und ermöglicht so die Theoriebildung komplementärer Muster. Beide Forschungsfragen führen über das hinaus, was diese Arbeit leisten kann - der Fokus hier liegt auf der detaillierten phonetischen Formbeschreibung und ihrer potenziellen Kontextabhängigkeit. Jedoch wird hierdurch weiterführenden Forschungsfragen die methodische und, da die Annotationsdaten größtenteils zur Verfügung gestellt werden dürfen, auch eine empirische Grundlage bereitet. Insgesamt führt der hier entwickelte Ansatz im besten Falle zu einer weniger holistischen und subjektiven Interpretation von Füllpartikeln und damit zu einer höheren Systematisierung der Füllpartikelforschung.

Um Form-Kontext-Muster zu finden, benötigt man eine Formalisierung der relevanten phonetischen Formen und linguistischen Kontexte. Tabelle 3.2 wiederholt das in Kapitel 2 erarbeitete Schema zur Darstellung der Formrepräsentationen von Füllpartikeln und erweitert es um ihre Kontextstruktur, die verschiedene Arten von Kontexten beschreibt, nämlich sequenzielle Kontexte (wie segmentale oder wortbasierte) und hierarchische Kontexte (wie phrasale, prosodische oder dialogstrukturelle), die ich in den folgenden Abschnitten 3.2.2 und 3.2.3 beschreibe und diskutiere.

In Tabelle 3.2 ist die orthographische und symbolphonetische Transkription von und ähm 〈Einatmen〉 zusammen mit ihren phonetisch-akustischen Beschreibungsparametern gegeben. Akustisch können mindestens die in Abschnitt 2.2 diskutierten phonetischen Parameter gemessen werden. Dabei wird die Füllpartikel in ihre kategorial trennbaren Laute segmentiert. Für verschiedene Segmente kann die Dauer 
Tabelle 3.2: Schema verschiedener Repräsentationsebenen von Füllpartikeln und ihrer Kontextstruktur anhand des fiktiven Beispiels und ähm, gefolgt von einem hörbaren Einatmungsgeräusch. Die akustischen Maßeinheiten werden in Kapitel 4 beschrieben. Die Füllpartikel ist in sequenzielle (se.) und hierarchische (hi.) linguistische Kontexte eingebettet.

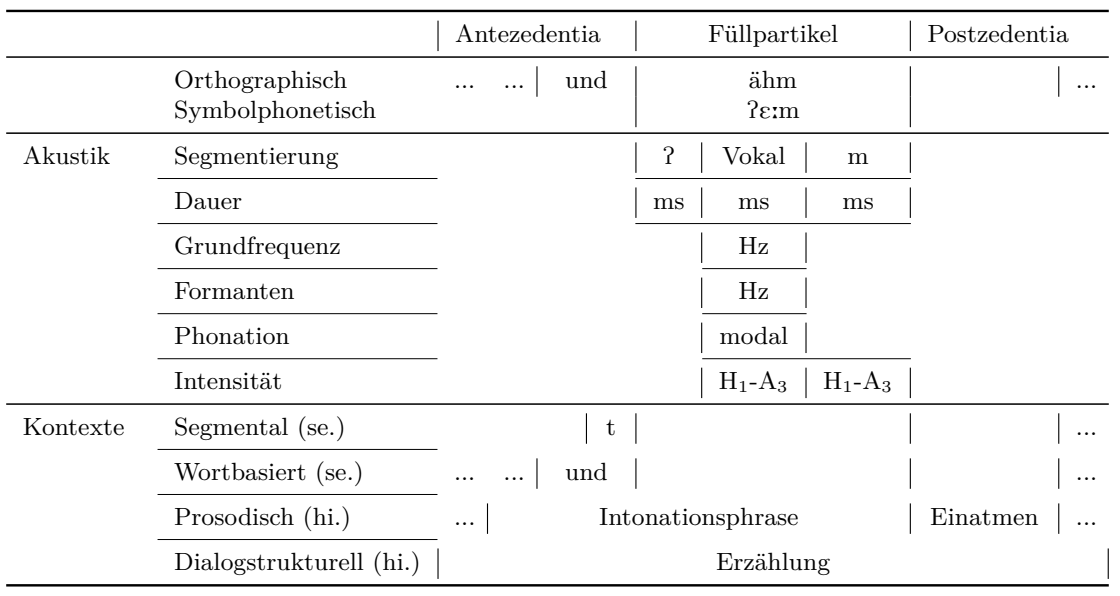

in Millisekunden, die Grundfrequenz und Formanten in Hertz und die Intensität in Dezibel gemessen werden. Die Phonation wird aufgrund methodischer Schwierigkeiten nur perzeptiv kategorisiert (hier als modal bezeichnet), vgl. Abschnitt 4.4. Der sequenzielle Kontext von $\ddot{a} h m$ besteht auf lautsegmentaler Ebene aus antezedentem [t] und postzedenter Pause, auf wortsegmentaler Ebene aus antezedentem und und postzedenter Pause. Der hierarchische Kontext besteht auf prosodischer Ebene aus einer Intonationsphrase, die aus und $\ddot{a} h m$ besteht und die vor dem Einatmungsgeräusch endet; auf dialogstruktureller Ebene ist und $\ddot{a} \mathrm{hm}$ in eine Erzählung eingebettet. Da Tabelle 3.2 zur besseren Darstellung ein fiktives Beispiel wiedergibt, werden die Kontexte nun nochmals kurz an einem tatsächlichen Beispiel aus dem Berlin Dialogue Corpus compilated v. $1\left(\mathrm{BeDiaCo}_{\mathrm{c}}\right)$ exemplifiziert. Beispiel (2) illustriert die verschiedenen Ausprägungen der sequenziellen und hierarchischen Auftretenskontexte und ihre Problematiken für einen Ausschnitt aus einem spontansprachlichen aufgabenfreien Dialog. ${ }^{66}$ Die bisherigen Erkenntnisse zu den jeweiligen Kontexten

\footnotetext{
${ }^{66}$ Ich gebe hier die ausprachebasierte Transliteration der diplomatischen Ebene in BeDiaCo wieder, da ich für jegliche Interpretation immer das akustische Signal hinzuziehe. Andere Transliterationsmöglichkeiten sind HIAT (Ehlich \& Rehbein 1976, 1979) oder GAT2 (Selting et al. 2009).
} 
werden anschließend ausführlicher in Abschnitt 3.2.2 und 3.2.3 zusammengefasst.

(2) a. A: $\langle\mathrm{P}, 961 \mathrm{~ms}\rangle$ äh wo kommst du ursprünglich her

b. B: aus $\mathrm{X}$ aus sonem $\langle\mathrm{P}, 372 \mathrm{~ms}\rangle$ ja aus kein Kuhkaff aber $\langle$ lacht $\rangle$

c. A: okay

d. B: also ne kleinere Stadt is das $\langle\mathrm{P}, 59 \mathrm{~ms}\rangle$ äh 〈Einatmung, $280 \mathrm{~ms}\rangle$ das kennt auch niemand

$(\mathrm{BeDiaCo}$ frei_f1f2_ch2, 257,6-267,3s)

Der sequenzielle segmentale Kontext von $\ddot{a} h$ in Beispiel (2a) besteht aus einer langen stillen Pausen von $961 \mathrm{~ms}$ und einem [v] in wo, der von äh in Beispiel (2d) besteht aus einer kurzen stillen Pause von 59 ms und einem Einatmungsgeräusch von 280 ms Dauer. Die Festlegung eines Schwellenwertes für die minimale Ausdehnung einer stillen Pause ist nicht trivial; kategorisiert man erst ab 250 ms eine stille Pause (Goldman-Eisler 1958, vgl. auch die Kritik von Hieke et al. 1983), so besteht der linkssegmentale Kontext von äh in Beispiel (2d) aus [s]. ${ }^{67}$ Allerdings führt die ,Wegkategorisierung، einer stillen Pause zu keiner Veränderung des akustischen Signals.

Der sequenzielle Wortkontext in (2a) besteht aus dem nachfolgenden Wort wo, der in (2d) aus das und das.

Der hierarchische syntaktische Kontext ist in den beiden Beispielen identisch mit den beiden Intonationsphrasen (IPn), es ist jedoch nicht eindeutig, ob $\ddot{a} h$ in die hierarchische Phrasenstruktur von also ne kleinere Stadt is das integriert wird oder ob es eine eigene Konstituente bildet (für eine syntaktische Analyse von $\ddot{a} h$ in hierarchischen Strukturen vgl. Ferreira \& Bailey 2004).

Der prosodische Kontext in (2a) ist nur hierarchisch zu fassen, als Einbettung in die IP äh wo kommst du ursprünglich her. In (2d) ist zu diskutieren, ob äh Bestandteil der IP also ne kleinere Stadt is das äh ist oder eine eigene IP konstituiert, da die Pause vor äh sehr kurz ist und die Intonationskontur der IP auf äh ohne Auffälligkeiten fortgeführt wird (vgl. Abschnitt 4.2.2).

Die dialogstrukturellen hierarchischen Kontexte lassen sich in unterschiedlicher Granularität und aus verschiedenen theoretischen Perspektiven beschreiben. Im Rahmen der Turn-Taking-Theorie (Sacks et al. 1974) müsste der Ausschnitt in Turns segmentiert werden. Während für Sprecherin A ein Turn in Beispiel (2a) relativ

\footnotetext{
${ }^{67}$ Die untere angenommene Grenze für stille Pausen wird in der Gesprochenen-Sprache-Forschung je nach Ziel der Studie und Hintergrund der Forschenden unterschiedlich gehandhabt und reicht von $10 \mathrm{~ms}$ bis $550 \mathrm{~ms}$ (vgl. den Überblick in Fors 2015: 40) oder gar bis $1000 \mathrm{~ms}$ (Leeuw 2007: 95).
} 
einfach und intuitiv als solcher zu erkennen ist und $\ddot{a} h$ hier demnach turn-initial stünde, müssten für die Analyse von Beispiel (2d) weitreichende Annahmen über Turns getroffen werden, die dann Voraussetzung für eine Segmentierung in entweder einen einzigen Turn ( $2 \mathrm{~b}$ mit $2 \mathrm{~d}$ ) oder zwei separate Turns (2b) und (2d) sind. In beiden möglichen Fällen stünde äh turn-medial, im zweiten Fall jedoch ,medialer (tatsächlich etwa in der Mitte des Turns) als im ersten Fall. Die Feststellung der Turn-Medialität ist also nur bedingt aussagekräftig und wird in dieser Arbeit nicht betrachtet.

Dialogstrukturell (Carletta et al. 1997, 1996) stünde äh in (2a) am Anfang einer W-Frage, äh in (2d) inmitten einer Antwort auf eine W-Frage. Auch in dieser Betrachtung existieren Segmentierungsprobleme, die sich auf die Interpretation der Füllpartikel auswirken. So ist beispielsweise unklar, ob der Satz das kennt auch niemand noch zur Antwort gezählt werden sollte oder nicht; falls der Satz als zusätzliche oder eigenständig motivierte Information betrachtet wird, stünde $\ddot{a} h$ am Ende der Antwort (zu dieser Diskussion vgl. Abschnitt 4.2.3).

Beispiel (2) und Tabelle 3.2 zeigen den Bedarf für eine systematische Analyse der Kontextstrukturen von Füllpartikeln. Auch wird gezeigt, dass eine solche Analyse keineswegs trivial ist und je nach Segmentierungsentscheidung verschiedene Ergebnisse zustande kommen. Mithilfe der Annotationsrichtlinien in Kapitel 4 wird versucht, dem entgegenzuwirken.

\subsubsection{Sequenzielle Kontexte}

In diesem Kapitel fasse ich Studien zu sequenziellen Füllpartikelkontexten auf segmentaler und wortbasierter Ebene zusammen, in welchen unterschiedliche phonetische Realisierungen beobachtet werden.

\subsubsection{Segmente}

Der segmentale Kontext beschreibt die den Füllpartikeln vorangehenden und nachfolgenden Segmente. Eine Sonderform phonetischer Segmente sind dabei stille Pausen (für einen Überblick zu stillen Pausen vgl. Fletcher 2010 und Fors 2015). Über Laute als segmentale Umgebung ist unabhängig von Füllpartikeln bekannt, dass sich in Konsonant-Vokal- (CV) und Vokal-Konsonant-Strukturen (VC) Transitionen in den Vokalformanten an den Übergängen von vorangehenden oder folgenden Kon- 
sonanten zeigen (Lehiste \& Peterson 1961). Dies sollte mutatis mutandis auch für Füllpartikeln gelten. Da jedoch über den lautsegmentalen Kontext von Füllpartikeln keine Studie auffindbar war, diskutiere ich nun ausgewählte Studien zu Füllpartikeln und stillen Pausen. Beide Kontexte, Laute und Pausen, untersuche ich auch in den beiden Korpusstudien (vgl. Kapitel 5 und 6).

Stille Pausen sind als Abwesenheit stimmhafter Komponenten im akustischen Signal definiert (vgl. Fletcher 2010: 573). Im Englischen ziehen Vokal-Nasal-Formen (VN-Formen) längere stille Pausen nach sich als dies bei vokalischen Formen (VFormen) der Fall ist (Clark \& Fox Tree 2002). Clark \& Fox Tree analysieren als Hauptquelle ihrer Studie das London-Lund-Korpus (Svartvik 1990), das aus 50 Gesprächen (170000 Wörter) britischer Erwachsener mit hauptsächlich akademischem Hintergrund besteht, die zwischen 1961 und 1976 aufgenommen wurden (Clark \& Fox Tree 2002: 80). Nach um sind Verzögerungen wie Pausen, Füllpartikeln und Pausen plus Füllpartikeln (ebd.: 83) sowohl signifikant häufiger (61\%) als nach uh $(29 \%)$, als auch signifikant länger (ebd.: 82). Die Pausendauer orientiert sich an den Transliterationen, die selbst wiederum eine kategorial-perzeptive Einschätzung seitens der Transkribierenden darstellen, keine akustische Messung. ${ }^{68}$ Da oft auch andere prosodische Signale wie beispielsweise Segmentlängungen als subjektive Pause perzipiert werden (Duez 1993), ist die Schlussfolgerung aus den Daten kritisch zu sehen. Vor um sind Verzögerungen signifikant häufiger (41\%) als vor uh (34\%), die Verzögerungsdauer ist hingegen nicht unterschiedlich lang. Hieraus folgern Clark \& Fox Tree, dass sich uh und um hauptsächlich darin unterscheiden, dass sie unterschiedlich lange Verzögerungen einleiten (Clark \& Fox Tree 2002: 86).

Ein ähnliches Ergebnis beschreibt die Studie von Smith \& Clark (1993), in der 25 englische Versuchspersonen je 40 Fragen beantworten, wobei 39mal uh und 38mal um produziert wird. Stille Pausen vor $u m$ sind im Mittel mit 1,1s länger als vor uh; Pausen nach um sind im Mittel 3,1s länger als nach uh (ebd.: 35, Tab.6). Die Position der Füllpartikel in der Antwort wird allerdings nicht näher beschrieben. Zudem handelt es sich um eine interviewähnliche Methode, die mit gleichberechtigten Dialogen nur schwerlich vergleichbar ist.

Rose (2015) untersucht eine Stichprobe von 165 min des Santa Barbara Corpus Teil

\footnotetext{
${ }^{68}$ „Brief pauses ,of one light foot ${ }^{6}$ are marked with periods (.), and unit pauses ,of one stress unit" with dashes (-). When we need a measure of pause length, we treat the unit pause as 1 unit long, and the brief pause as 0.5 units long [...]. (Clark \& Fox Tree 2002: 80).
} 
1 (Du Bois et al. 2000) mit 149 Füllpartikeln in aufgabenfreien Alltagssituationen, die nicht im Labor stattfinden. Während sich $u$ und um nicht signifikant in der Dauer unterscheiden, folgen jedoch nach um mit 36,9\% der Fälle signifikant häufiger stille Pausen nach als nach $u h(16,9 \%)$. Die mittlere Dauer der stillen Pause ist nach um (+ $910 \mathrm{~ms})$ nur marginal länger als nach $u h(+744 \mathrm{~ms})$. Rose fasst die Dauer der V/VN-Form und die Dauer der nachfolgenden stillen Pause unter dem Maß des „total delay" zusammen. Für VN + stille Pause ist die Gesamtdauer signifikant länger als für $\mathrm{V}+$ stille Pause (Rose 2015: 74).

Jessen (2012) fasst unveröffentlichte Ergebnisse ${ }^{69}$ zu Füllpartikeln in segmentalen Kontexten in deutscher Spontansprache („erschwerte Bildbeschreibung“, ebd.: 158) von 72 Männern danach zusammen, ob vor oder nach einem äh oder einem ähm eine stille Pause (in seiner Studie alles über $100 \mathrm{~ms}$, ebd.: 148) steht, oder ob sich die Füllpartikel (FP) „nahtlos an den vorangehenden bzw. folgenden Kontext anschließt“" (ebd.: 149). Jessen gibt die prozentuale Verteilung von allen $\ddot{a} h$ - und $\ddot{a} h m$-Entitäten für die vier Kontexte Laut-FP-Laut, Pause-FP-Laut, Laut-FP-Pause und Pause-FPPause wieder (ebd.: 154). 41,6\% aller ähm stehen zwischen Pausen, aber nur 19,6\% aller $\ddot{a} h$, während $37 \%$ aller $\ddot{a} h$ zwischen Wörtern stehen, aber nur 16,4\% aller ähm (meine Berechnungen). ${ }^{70}$ Jessen schließt daraus, dass der „Typ äh [...] also meistens in einen lautlichen Kontext eingebettet [ist], während [der] Typ ähm meistens isoliert in Erscheinung [tritt]“. Diese Verteilung spiegelt die Tendenz der Studie von Clark \& Fox Tree (2002) wider. Clark \& Fox Tree stellen zusätzlich die Hypothese auf, dass VN-Formen eine längere nachfolgende Pause anzeigen als V-Formen. Dies ist aus den Daten von Jessen nicht zu entnehmen, allerdings sind beide Formen in Pause-FPPause-Kontexten länger $\left(\bar{x}_{\ddot{a} h}=447 \mathrm{~ms}, \mathrm{~s}=152 \mathrm{~ms} ; \bar{x}_{\ddot{a} h m}=560 \mathrm{~ms}, \mathrm{~s}=202 \mathrm{~ms}\right)$ als in Laut-FP-Laut-Kontexten $\left(\bar{x}_{\ddot{a} h}=317 \mathrm{~ms}, \mathrm{~s}=132 \mathrm{~ms} ; \bar{x}_{\ddot{a} h m}=443 \mathrm{~ms}, \mathrm{~s}=141 \mathrm{~ms}\right)$ (Jessen 2012: 154).

Sowohl fürs Englische als auch fürs Deutsche lässt sich also tentativ sagen, dass VN-Formen häufiger zwischen stillen Pausen stehen als V-Formen, während sich

\footnotetext{
${ }^{69}$ Basierend auf Studien von Trouvain, Tschäpe et al. (2005) und einer unveröffentlichten Magisterarbeit von Bauer (2007): Sprecherspezifik der Stimmtonhöhe in gefüllten Pausen und am Ende von Intonationsphrasen. Magisterarbeit, Universität des Saarlandes, Fach Phonetik und Phonologie.

${ }^{70}$ Tabelle 5-2 in (Jessen 2012: 154) gibt Prozentzahlen aller äh und ähm gemeinsam auf alle Kontexte verteilt wieder, d. h. alle Zahlen zusammen ergeben $100 \%$. Allerdings kommt $\ddot{a} h$ in $68,3 \%$, ähm nur in $31,7 \%$ aller Fälle vor. Zur Evaluierung der Vorkommen je äh- und ähm-Gesamtheit für jeden Kontext normalisiere ich die prozentuelle Verteilung je Form, so dass jede Formspalte $100 \%$ ergibt.
} 
das Verhältnis in Laut-FP-Laut-Kontexten eher umkehrt. Auch ist die Dauer einer Füllpartikel zwischen zwei Pausen länger als in anderen Kontexten, während für die Länge der Pause im Zusammenhang mit der Länge der Füllpartikel keine konsistenten Ergebnisse vorliegen.

\subsubsection{Wörter}

Dieser Abschnitt betrachtet nicht mehr die Ebene der füllpartikeladjazenten Laute, sondern die der füllpartikeladjazenten Wörter, vergrößert also den Kontext. Auch in dieser Ebene werden Pausen betrachtet, auch wenn sie nun nicht mehr mit Lauten, sondern eben mit Wörtern verglichen werden. Die Vergleichbarkeit ist in diesem Fall dennoch gegeben, da Laute im vorherigen Abschnitt meistens auch Anfänge oder Enden von Wörtern darstellen.

Analog und zu den oben beschriebenen Pausen- und Laut-Kontexten in Jessen (2012) analysiert Leeuw (2007) in Interviews mit 21 deutschsprachigen Versuchspersonen Pausen- und Wort-Kontexte (vgl. Tabelle 3.3). Nur Pausen, die länger als eine Sekunde waren, gehen in die Kategorie Pause ein, kürzere Dauern sind der Kategorie Wort zugeordnet (ebd.: 95). VN-Formen erreichen im Vergleich zu Vund N-Formen die höchsten prozentualen Werte besonders in Wort-FP-Pause- und Pause-FP-Wort-Kontexten. Trotz ihrer ähnlichen Vorgehensweise lassen sich diese Kontexte jedoch nicht mit den von Jessen (2012) ermittelten Werten vergleichen, da Jessen alle Kontexte und Formen zu $100 \%$ addiert, während Leeuw die Formen je Kontext zu $100 \%$ addiert und sich die Pausenschwellen stark unterscheiden (100 ms bei Jessen vs. $1000 \mathrm{~ms}$ bei Leeuw).

Tabelle 3.3: Vergleich der Anteile vokalischer, vokalisch-nasaler und nasaler Füllpartikelformen bei deutschsprachigen Versuchspersonen in Wort-Pause-Kontexten in der Studie von (Leeuw 2007: 104).

\begin{tabular}{l|ccc}
\hline & \multicolumn{3}{|c}{ Füllpartikelform } \\
Kontext & vokalisch & vokalisch-nasal & nasal \\
\hline Wort-FP-Wort & $42 \%$ & $58 \%$ & - \\
Wort-FP-Pause & $17 \%$ & $82 \%$ & $1 \%$ \\
Pause-FP-Wort & $6 \%$ & $75 \%$ & $19 \%$ \\
Pause-FP-Pause & $27 \%$ & $58 \%$ & $15 \%$ \\
\hline
\end{tabular}

Zu Wörtern beziehungsweise Wortarten in der Umgebung von Füllpartikeln exis- 
tieren einige weitere Studien, die allerdings nur vereinzelt die phonetische Form von Füllpartikeln betrachten und eher auf Wortarten abzielen. ${ }^{71}$

Ob nun von Laut- oder Wortkontexten die Rede ist, ist also für die phonetische Form vorerst nicht von Bedeutung, da aufgrund inkonsistenter beziehungsweise im Deutschen nicht vorhandener Ergebnisse zu Wortarten keine Aussage getroffen werden kann.

\subsubsection{Hierarchische Kontexte}

Mit hierarchischen Kontexten beschreibe ich alle Kontexte, innerhalb derer weitere, über laut- und silbensegmentale Einheiten hinausgehende hierarchische Abhängigkeiten bestehen können, wie syntaktische Phrasen, Intonationsphrasen, Äußerungen, Turns und dialogstrukturelle Einheiten. Dieser Abschnitt diskutiert die bisher beobachteten unterschiedlichen phonetischen Realisierungen in solchen Kontexten. Bisweilen ist nicht klar, ob eine Füllpartikel, die am Rand einer solchen Konstituente steht, auch Teil der Konstituente ist. In solchen Fällen würde auch eine sequenzielle Betrachtung infrage kommen, da die Füllpartikel dann zwischen zwei Einheiten stünde. Daher werde ich in diesem Kapitel auch Studien diskutieren, die solche Positionen am Rande von größeren Konstituenten erwähnen, ohne tiefergehend die genaue theoriegeleitete Einbettung in ebendiese Strukturen zu diskutieren.

\subsubsection{Syntaktische Phrasen und Äußerungen}

Shriberg (1994) untersucht drei Korpora des amerikanischen Englisch. ${ }^{72}$ In allen drei Korpora tendieren V-Formen eher dazu, satzmedial aufzutreten und VN-Formen eher dazu, satzinitial aufzutreten (ebd.: 154). Über alle Korpora hinweg liegt der Anteil satzinitialer Füllpartikeln an allen vorkommenden Füllpartikeln bei 41,5\%$64,3 \%$, der Anteil satzmedialer Füllpartikeln entsprechend bei 35,7\%-58,5\% (ebd.: 154-155). In allen drei Korpora ist die V-Form häufiger als die VN-Form (ebd.: 155).

\footnotetext{
${ }^{71}$ Fürs Englische beschreiben Maclay \& Osgood (1959) \& Martin \& Strange (1968), dass Füllpartikeln eher vor Inhalts- als vor Funktionswörtern zu finden sind. Andere Studien finden keinen Unterschied (Beattie \& Shovelton 2002; Cook 1971; Rose 2015) oder mehr Füllpartikeln vor Funktionswörtern (Hawkins 1971) oder mehr Füllpartikeln nach Funktionswörtern (Schneider 2014).

${ }^{72} 66$ Sprecherinnen und Sprecher mit Reiseplanungsdialogen in AMEX (ca. 12000 Wörter, Kowtko \& Price 1989), 15 Sprecherinnen und 15 Sprecher aufgabenfreier Telefongespräche in Switchboard (ca. 40000 Wörter, Godfrey \& Holliman 1993), und Mensch-Computer-Dialoge aus ATIS (D. A. Dahl et al. 1994) mit ca. 180000 Wörtern (Shriberg 1994: 35-40).
} 
Satzinitiale Disfluencies (hierunter definiert Shriberg nicht nur Füllpartikeln) werden wahrscheinlicher, je länger ein Satz ist (Shriberg 1994: 106-107). Auch Schneider (2014) findet für einen Teil des Switchboard Corpus des Amerikanischen Englisch, dass besonders am Beginn oder in der Nähe des Beginns von Sätzen eine ,Häsitation` (in dieser Studie „filled and unfilled pauses“, ebd.: 1) produziert wird (ebd.: 243), was sich mit den Ergebnissen von Shriberg (1994) deckt. Die Formen uh und uhm haben ein ähnliches Verteilungsmuster innerhalb von Präpositionalphrasen (Schneider 2014: 63), eine genaue Aufschlüsselung der Formvarianten über die Positionen zu Beginn von Sätzen wird jedoch nicht gegeben.

O'Shaughnessy (1992) untersucht Mensch-Computer-Interaktionen, in denen 42 Versuchspersonen dem Computer Anweisungen zur Reiseplanung geben. ${ }^{73}$ Er unterscheidet ,grammatische ${ }^{6}$ Füllpartikeln an „major syntactic boundaries" von ,ungrammatischen“ Füllpartikeln „within small syntactic units, such as noun groups“ (ebd.: I-521). Die erste Kategorie zeigt eine Dauer von 200-500 ms, die zweite Kategorie eine Dauer von 170-320 ms. Auch hier wird keine Unterscheidung der phonetischen Realisierungen vorgenommen.

Zur Dauer der Einzelformen findet Rose (2015) in der oben schon erwähnten Stichprobe des Santa Barbara Corpus, dass die mittlere Dauer von uh und um an größeren Grenzen wie Diskurs- und Satzgrenzen kürzer [sic!] ist $\left(\bar{x}_{u h}=552 \mathrm{~ms}\right.$, $\bar{x}_{u m}=680 \mathrm{~ms}$ ) als an kleineren Grenzen wie dem Beginn subordinierender Sätze oder Präpositionalphrasen $\left(\bar{x}_{u h}=641 \mathrm{~ms}, \bar{x}_{u m}=963 \mathrm{~ms}\right)$ (ebd.: 75). Dieses Ergebnis steht konträr zu den Ergebnissen von O'Shaughnessy (1992).

Zusammenfassend zeigen die Studien, dass Füllpartikeln häufiger an syntaktischen Konstituentengrenzen auftreten als innerhalb von Konstituenten, insbesondere an oder in der Nähe von Satzgrenzen. Bisher wird jedoch nur in wenigen Studien Hinweisen auf unterschiedliche phonetische Realisierungsformen in diesen syntaktischen Kontexten nachgegangen. In englischen Dialogen kommen VN-Formen satzinitial häufiger vor als satzmedial, für kleinere Konstituenten wie beispielsweise Präpositionalphrasen wird kein Formhäufigkeitsunterschied festgestellt. ${ }^{74}$ Die Ergebnisse

\footnotetext{
${ }^{73}$ Die untersuchte Sprache ist sehr wahrscheinlich Englisch in Kanada. Von 36 Sprecherinnen und Sprechern wurden jeweils die ersten drei Äußerungen in diesem experimentellen Setting analysiert, hinzu kommen nochmal je drei Äußerungen von sechs zusätzlichen Sprecherinnen und Sprechern, die Pausen enthielten.

${ }^{74}$ Ähnliche Ergebnisse finden sich auch in monologischen Nacherzählungen. Fraundorf \& Watson (2013) untersuchen 15 englische Versuchspersonen zwischen 18 und 22 Jahren, die drei Passagen
} 
zur Dauer von Füllpartikeln an unterschiedlichen Positionen sind inkonsistent. Syntaktische Phrasen werden in dieser Arbeit daher nicht weiter betrachtet.

\subsubsection{Intonationsphrasen}

Eine Intonationsphrase (IP) ist eine prosodische Konstituente beziehungsweise prosodisch definierte Phrase, ,which [...] is the domain of a perceptually coherent intonational contour, or tune“ (Shattuck-Hufnagel \& Turk 1996: 210). Im Rahmen der prosodischen Planung einer Äußerung kann eine syntaktische Konstituenten- oder Satzgrenze gleichzeitig auch eine Intonationsphrasengrenze darstellen (Ferreira 1993; Krivokapić 2007; für einen Überblick vgl. Fletcher 2010). Andererseits können syntaktische und prosodische Grenzen auch auseinanderfallen (vgl. Shattuck-Hufnagel \& Turk 1996: 200). Intonationsphrasen werden durch verschiedene phonetische Merkmale markiert, vgl. Vaissière (1983), Peters et al. (2005) und Abschnitt 4.2.2.

An Intonationsphrasengrenzen und innerhalb von Intonationsphrasen können Füllpartikeln stehen. Clark \& Fox Tree (2002) definieren in ihrer Korpusstudie drei Positionen an und in Intonationsphrasen, die sie aus sukzessive geringeren Anforderungen an die Sprachplanung ableiten: „(I) at the boundary; (II) after the first word (ignoring $u h$ and $u m$ ); and (III) later" (ebd.: 94). Sie beziehen die Möglichkeit einer Füllpartikel an Position I-III auf die Wortlänge einer IP und ermitteln signifikant unterschiedliche Füllpartikelraten von 4,3\% für Position I, von 2,7 \% für Position II und von 1,3\% für Position III; d. h. Füllpartikeln sind in Position III weniger wahrscheinlich, obwohl es in dieser Position mehr Möglichkeiten für sie gäbe, aufzutreten. $56 \%$ der Füllpartikeln in Position I, aber nur $39 \%$ beziehungsweise $37 \%$ der Füllpartikeln in den Positionen II und III sind VN-Formen (ebd.). VN- und V-Formen stehen demnach in diesem Korpus etwa gleich häufig an der Grenze, also vor einer IP. Nach dem ersten Wort und innerhalb einer IP überwiegen die V-Formen.

Swerts (1998) untersucht mithilfe von zwölf monologischen Bildbeschreibungen zweier Sprecherinnen im Niederländischen (insgesamt 46,5 min und 310 Füllpartikeln, ebd.: 487, also 6,7 FP/min), ob „FPs [filled pauses - M. B.] may be informative

aus Alice im Wunderland anhand einer zusätzlichen Liste mit plot points nacherzählen sollen, die sie nach dem Lesen des Textes erhalten. Sowohl der Beginn einer Äußerung, der Beginn einer grammatischen Phrase, der Beginn einer Reparatur und der Beginn eines neuen Plots erhöhen die Wahrscheinlichkeit, dass eine Füllpartikel auftritt, signifikant (Fraundorf \& Watson 2013: 1089). Äußerungen sind definiert als ,subject and predicate together separated by a discernible prosodic break" (ebd.: 1087). 
about [...] units that exceed the level of the sentence" (Swerts 1998: 486). Genauer untersucht Swerts, ob die strukturellen Eigenschaften eines Diskurses die Distribution von (bestimmten) Füllpartikeln voraussagen können (ebd.: 487). Hierzu lässt er die zwölf erhobenen Monologe von 19 Annotatoren anhand von Pausen, Tonhöhenreset, Grenztontyp und weiterer, nicht näher benannter Parameter in „prosodische Phrasen" segmentieren (ebd.: 487 f.), um auf diese Weise Einblicke in die Verteilung von Füllpartikeln an den Rändern und in der Mitte dieser Phrasen zu erhalten. Phrasengrenzen, die von über $75 \%$ der Annotatoren als Grenze markiert werden, definiert Swerts als ,stark', die anderen als ,schwach' (ebd.: 488). Ausgewertet werden initiale und mediale Positionen; mögliche finale Positionen werden als initiale kategorisiert (ebd.). Von 502 IPs kommen in 226 IPs Füllpartikeln vor (45\%), davon enthalten 109 IPs mediale Füllpartikeln, 117 initiale Füllpartikeln. Sieben der 109 Füllpartikeln in medialen IPs werden als ,stark ${ }^{6}$ kategorisiert; von den initialen sind es 43 der 117. Von allen 64 als stark kategorisierten Grenzen enthalten demnach 50 Stück $(78 \%$ ) eine Füllpartikel (ebd.). Swerts schließt daraus, dass eine IP-Grenze die Wahrscheinlichkeit für Füllpartikeln erhöht (ebd.). Diese Schlussfolgerung ist kritisch zu sehen - ebenso denkbar ist, dass das Vorkommen einer Füllpartikel von den Annotatoren als Hinweis für eine IP-Grenze interpretiert wurde.

In initialer Position sind $75 \%$ aller Füllpartikeln VN-Formen und $25 \%$ V-Formen ( in toto $n=117$ ), in medialer Position sind $12 \%$ aller Füllpartikeln VN-Formen und $88 \%$ V-Formen (in toto $n=193$ ) (ebd.: 490). Obwohl unklar bleibt, ob die Vergleichbarkeit von Swerts' medialer Position zu den IP-Positionen II und III von Clark \& Fox Tree (2002) gegeben ist beziehungsweise ob die Kategorien „prosodische Phrase“ und „Intonationsphrase“ übereinstimmen, lässt sich doch für den Beginn von diesen prosodisch orientierten Phrasen eine ähnliche Tendenz feststellen, nämlich dass VNFormen mit $75 \%$ bei Swerts 1998 und $56 \%$ bei Clark \& Fox Tree 2002 überwiegen (allerdings mit der Unschärfe, dass die Einteilung bei Swerts auch potenziell finale Füllpartikeln in dieser Position enthält, s. oben). Füllpartikeln in initialer Position sind zudem signifikant länger $(\bar{x}=58,7 s)$ als in medialer Position $(\bar{x}=36,2 s)$ (Swerts 1998: 491).

Tabelle 3.4 zeigt, dass Kontexte mit vorangehender Pause und beidseitiger Pause in der Studie von Swerts (ebd.) eher symptomatisch für initiale Positionen und Kontexte mit vorangehendem Wort oder beidseitigem Wort eher symptomatisch 
für mediale Positionen sind. ${ }^{75}$ Dies ist nicht allzu verwunderlich, da einerseits das Auftreten stiller Pausen ein möglicher Hinweis auf die Grenzen einer prosodischen Phrase sein kann (vgl. Vaissière 1983) und es andererseits in medialer Phrasenposition aufgrund ihrer potenziell längeren Spanne eine höhere Wahrscheinlichkeit für Wort-FP-Wort- und Wort-FP-Pause-Kontexte gibt. In initialer Position zeigen die niederländischen Füllpartikeln eine signifikant höhere Tonhöhe und eine signifikant längere Dauer als in medialer Position (Swerts 1998: 491).

Tabelle 3.4: Vergleich der Anteile initialer und medialer Füllpartikeln in prosodischen Phrasen (PP) bei niederländischsprachigen Versuchspersonen in Wort-Pause-Kontexten in der Studie von Swerts (1998: 491) (Berechnung von mir - M. B.).

\begin{tabular}{l|cc}
\hline & \multicolumn{2}{|c}{$\begin{array}{c}\text { Position in der prosodischen Phrase } \\
\text { initial }\end{array}$} \\
Kontext & $10 \%(n=7)$ & $90 \%(n=66)$ \\
\hline Wort-FP-Wort & $10 \%(n=11)$ & $90 \%(n=94)$ \\
Wort-FP-Pause & $69 \%(n=33)$ & $31 \%(n=15)$ \\
Pause-FP-Wort & $69 \%(n=66)$ & $21 \%(n=18)$ \\
Pause-FP-Pause & $79 \%(n)$ \\
\hline
\end{tabular}

Von 204 „Häsitationspartikeln wie äh und ähm" (Peters 2005b: 310) an turninternen Phrasengrenzen im Lindenstraßenkorpus (sechs aufgabenbasierte Dialoge von vier männlichen und acht weiblichen deutschsprachigen Versuchpersonen, 80 Minuten, ca. 13000 Wörter, vgl. Peters 2005a) zeigt der größte Teil (80,9\%) nur eine schwache intonatorische Bewegung, die zumeist als eben oder leicht fallend kategorisiert wird (Peters 2005b: 312). Von den Füllpartikeln mit starken melodischen Ausprägungen sind 26 Stück stark fallend. Nur 8 Stück werden als fallend-steigend und nur 3 Stück als stark steigend klassifiziert (ebd.). Peters analysiert Füllpartikeln augenscheinlich nur an Phrasenenden und nicht an anderen Positionen (ebd.: 206).

Die Betrachtung des prosodischen Kontext der Intonationsphrase zeigt also nicht nur die Distribution von Füllpartikeln (sie sind zu Beginn von IPs wahrscheinlicher als später in der IP) sondern auch den Zusammenhang mit der realisierten phonetischen Form von Füllpartikelformen. So sind VN-Formen IP-initial im Englischen und Niederländischen häufiger als V-Formen; in nicht-initialen Positionen dreht sich das Verhältnis um. Füllpartikeln zeigen eine höhere Grundfrequenz $\left(f_{0}\right)$ und eine längere Dauer IP-initial im Englischen im Vergleich zu IP-medial (Swerts 1998) sowie eine eher ebene, fallende oder stark-fallende IP-finale Intonationskontur im Deutschen

\footnotetext{
${ }^{75}$ Über den unteren Schwellenwert einer stillen Pause gibt Swerts (1998) keine Auskunft.
} 
(Peters 2005b). Diese Merkmale passen zu den prosodische Merkmalen von Intonationsphrasen, die im Allgemeinen eine absinkende $f_{0}$ über die Dauer einer IP aufweisen (vgl. Vaissière 1983). Überhaupt ist zu erwarten, dass sich prosodische Effekte auf die Realisierung der phonetischen Füllpartikelform auswirken, wie beispielsweise Sprechgeschwindigkeit, finale Längung und initiale Stärkung von Segmenten an Phrasenenden (für einen Überblick s. Cho 2015). Diese prosodischen Effekte werden aber meines Wissens nicht im Zusammenhang mit Füllpartikeln berücksichtigt. So hat der Beginn einer Intonationsphrase eine verstärkende Wirkung auf die phonetischen Merkmale von Segmenten an dieser Position (initiale Stärkung). Beispielsweise wird der Vokal /a/ im Amerikanischen Englisch zu Beginn von Intonationsphrasen länger artikuliert als zu Beginn von (hierarchisch niedrigeren) Wortgrenzen (Cho 2008). Auch in intonationsphrasenfinaler Position zeigen sich Effekte, insbesondere Längungseffekte (finale Längung). Lange (gespannte) und kurze (ungespannte) Vokale in Silbennuklei sowie Nasale in Silbenkodas, wie sie in VN-Formen vorkommen, werden in einsilbigen deutschen Wörtern in IP-finaler Position signifikant länger produziert als in IP-medialer Position (vgl. Belz et al. [eingereicht]). Die längere Dauer einer Füllpartikel könnte also zumindest in Teilen durch ihre Position zu Beginn oder Ende einer IP und damit durch das Wirken initialer Stärkung oder finaler Längung auf ihre Segmente erklärt werden (was sich letztlich auch gezeigt hat, vgl. Abschnitt 6.2.3).

\subsubsection{Dialogstruktur}

Über Intonationsphrasen lassen sich verschiedene weitere Strukturen beobachten: Äußerungen, Redebeiträge (Turns) und Dialogstruktur, deren Argumentbereiche sich teilweise überschneiden. In dieser Arbeit untersuche ich die Einheit mit dem maximalen Ausdehnungspotenzial in dyadischen Dialogen, nämlich Dialogstruktur. ${ }^{76}$ Als Dialog verstehe ich nach Imo den

interaktive[n] Austausch von mindestens zwei Personen [...], die von Angesicht zu Angesicht, zeitlich und räumlich kopräsent, mit verbalen, paraverbalen und nonverbalen Mitteln kommunizieren (Imo 2016: 341 f.).

\footnotetext{
${ }^{76}$ Ich vermeide den Terminus Diskurs, da sich damit vielfältige andere Forschungsrichtungen verbinden (Grosz et al. 1995; Kamp et al. 2011; Mann \& Thompson 1988; Stutterheim 1997).
} 
Das Verständnis von Dialog in dieser Arbeit ist demnach der direkte interaktive verbale Austausch von zwei zeitlich und räumlich kopräsenten Personen von Angesicht zu Angesicht. Ferner wird nur die aufgabenfreie Variante eines spontansprachlichen Dialogs berücksichtigt.

Arbeiten zur Untersuchung von Dialogstruktur existieren hauptsächlich als überwiegend qualitative Studien innerhalb der Konversationanalyse (Goodwin 1981; Schegloff et al. 1977) und der Interaktionalen Linguistik (Selting 2007). Grundlegende Arbeiten für die quantitativ-qualitative Beschreibung von dialogischen Strukturen sind die Studien von Carletta et al. (1997, 1996). Die dort entwickelten Schemata legen zugrunde, dass sich Dialoge sequenziell-interaktional entwickeln, und dass sich diese Strukturen beschreiben lassen. Sie basieren auf der Theorie zu ,Dialogspielen („dialogue games“ in der gleichnamigen Monographie von Carlson 1985). Dialogspiele bestehen aus ,utterances starting with an initiation and encompassing all utterances up until the purpose of the game has been either fulfilled [...] or abandoned" (Carletta et al. 1997: 14). Somit ist der Begriff des Dialogspiels als ein übergeordnetes Thema zu verstehen, welches im Dialog behandelt wird. Dialogspiele bestehen wiederum aus Sequenzen von Dialogzügen (Engl. ,dialogue moves $\left.{ }^{6}\right),{ }^{77}$ „which are [...] initiations and responses classified according to their purposes" (ebd.). Mit Initiierungen und Antworten sind beispielsweise Dialogzüge gemeint, in denen eine Frage gestellt wird (initiativer Dialogzug, s. Beispiel 3a), die dann vom Gegenüber beantwortet wird (responsiver Dialogzug, s. Beispiel 3b). Dialogzüge (DZ) stellen also gewissermaßen Dialogstrukturkonstituenten dar.

(3) a. A: Wie findest du das Essen in der Mensa?

b. B: äh ich hab $\langle\mathrm{P}, 180 \mathrm{~ms}\rangle$ hier im Campus no nie in der Mensa gegessen weil ich eignlich in $\mathrm{X}$ bin

$(\mathrm{BeDiaCo}$ : a_f $8 \mathrm{f} 9,5,6-11,6 \mathrm{~s})$

Ich werde im Folgenden die Ergebnisse von Studien zu Füllpartikeln in dialogstrukturellen Kontexten wiedergeben und anschließend die sich daraus ergebende weitere Fragestellung diskutieren. Die bisherige Forschung konzentriert sich besonders auf das Auftreten von Füllpartikeln in bestimmten Positionen des Dialogs ohne Beschreibung der phonetischen Form.

Lickley (2001) untersucht Füllpartikeln im aufgabenbasierten Map-Task-Korpus

\footnotetext{
${ }^{77}$ Dialogue move wird synonym zu conversational move verwendet (Carletta et al. 1997: 19).
} 
HCRC (Anderson et al. 1991) fürs Englische. ${ }^{78}$ Er annotiert Dialogzüge nach dem Dialogstrukturannotationsschema von Carletta et al. (1997, 1996). Im Korpus sind 1492 Füllpartikeln enthalten (Lickley 2001: 94), was ca. einem Prozent aller Wörter entspricht. Am häufigsten sind Füllpartikeln in Antworten auf W-Fragen (ca. $15 \%$ ) und in instruierenden Zügen (ca. 12\%) enthalten, bei einer mittleren Wortlänge der Züge von 8,2 (vgl. Abb. 2, ebd.: 95). Responsive Dialogzüge wie Antworten auf W-Fragen und negative Antworten auf Entscheidungsfragen zeigen sowohl insgesamt als auch in einem Subset von 4-6 Wörtern je Dialogzug mit die höchsten Füllpartikelraten (ca. 1,75-2,5 je 100 Wörter, vgl. Abb. 3, ebd.). Erklärt wird dies damit, dass in diesem aufgabenbasierten Format Bestätigungen, wie sie in positiven Antworten auf Entscheidungsfragen vorkommen, weniger aufwendig zu planen sind, während für negative Antworten und komplexe Antworten auf W-Fragen ein höherer Suchaufwand in der Map Task angenommen werden kann (ebd.: 96).

Nicholson et al. (2010) testen für aufgabenbasierte Dialogen von drei Sprechern und drei Sprecherinnen (sechs gleichgeschlechtliche Dialoge) des amerikanischen Englisch im Korpus Indiana CReST (Eberhard et al. 2010), ob responsive Züge mehr Füllpartikeln enthalten als initiierende Dialogzüge. Sie nutzen die Dialogzugkategorisierung von Carletta et al. (1997). Initiierende Züge enthalten „Instructions, Explains, or Queries“, responsive Züge enthalten Antworten auf Fragen, „[a]cknowledgments that a previous move was heard and understood, or a clarification of an answer" (Nicholson et al. 2010: 90). Über die Segmentierung wird nichts gesagt. Eine Unterscheidung nach Position in einer ,Äußerung' wird auch vorgenommen, nach einer Definition, die sowohl Position I also auch Position II nach Clark \& Fox Tree (2002) entspricht (vgl. Abschnitt 3.2.3.2). ${ }^{79}$ Füllpartikeln sind in initiierenden Zügen signifikant häufiger als in responsiven Zügen (Nicholson et al. 2010: 91), was demnach nicht zu den Ergebnissen der Studie von Lickley (2001) passt.

Die Berücksichtigung der Dialogzüge bringt also mehr Wissen um die dialogstrukturellen Besonderheiten dyadischer Kommunikation in die Positionsanalyse von Füllpartikeln mit ein, als es eine rein prosodische oder eine übergeordnete turnsequenzielle Betrachtung könnte. Zur Veranschaulichung möchte ich nochmals kurz auf

\footnotetext{
${ }^{78} 128$ Dialoge mit 64 schottischen Studierenden, insgesamt 15 Stunden Sprachmaterial und ca. 150000 Wörter (Lickley 2001: 94).

${ }^{79}$ "A FP was deemed utterance-initial if it was the first word in a move or if it was preceded only by a single discourse marker (and, so, now). All other FPs were considered utterance-medially." (Nicholson et al. 2010: 90).
} 
Beispiel (3) zurückkommen, hier wiederholt als Beispiel (4). Am Anfang des beantwortenden Dialogzugs von Person B in (4b) steht ein $\ddot{a} h$. Intonationsphrasen werden in runden Klammern mit subskribiertem IP markiert, Dialogzüge mit Raute und subskribiertem DZ. Verschiedene Segmentierungen in unterschiedlichen Granularitätsstufen führen zu einer unterschiedlichen Anzahl an zu analysierenden Kategorien. Auf diese Weise enthält Beispiel (4) 12 Turns/Sprecherwechsel (6 von A, 6 von B), 9 Dialogzüge (5 von A, 4 von B) und 17 Intonationsphrasen (10 von A, 7 von B). Intonationsphrasengrenzen können mit Dialogzügen beziehungsweise Konstituenten der Dialogstruktur zusammenfallen, genauso wie syntaktische Grenzen mit Intonationsphrasen zusammenfallen können (für Diskussionen zu den Annotationsrichtlinien vgl. Abschnitt 4.2.3 für Dialogzüge und Abschnitt 4.2.2 für Intonationsphrasen).

(4) a. A: \#(Wie findest du das Essen in der Mensa?) IP \# DZ Initiierung Frage

b. B: \#(äh ich hab $\langle\mathrm{P}, 180 \mathrm{~ms}\rangle$ hier im Campus no nie in der Mensa gegessen weil ich eignlich in beep bin $)_{\mathrm{IP}}$

c. A: \#(mhm) $)_{\text {IP }} \#$ DZ Feedback

d. B: (an dem Campus dort) $)_{\mathrm{IP}}$

e. A: \#(mhm) $)_{\text {IP }} \#_{\text {DZ Feedback }}$

f. B: (deshalb) IP $\langle\mathrm{P}, 860 \mathrm{~ms}\rangle$ (das bei uns is ziemlich gut aber ich hör immer dass so generell die Leute nich so zufrieden sin mit den Mensen) IP \# DZ Antwort

g. A: \#(mhm) $)_{\text {IP }} \#_{\text {DZ Feedback }}$

h. $\quad \#(\text { also ich bin sehr })_{\mathrm{IP}}\langle\mathrm{P}, 340 \mathrm{~ms}\rangle$ (positiv überrascht) ${ }_{\mathrm{IP}}$

i. B: \#(ja?) $)_{\text {IP } \# \text { DZ Feedback }}$

j. A: (von der Mensa) IP (also ich hab davor mein Bachelor in beep gemacht) ${ }_{\text {IP }}$

k. B: \#(mhm) $)_{\mathrm{IP}} \#_{\mathrm{DZ}}$ Feedback

1. A: (un da is die Mensa) $)_{\mathrm{IP}}\langle\mathrm{P}, 286 \mathrm{~ms}\rangle$ (überhaupt nich gut) ${ }_{\mathrm{IP}} \#_{\mathrm{DZ}}$ Initiierung

m. B: \#(okay) ${ }_{\mathrm{IP}} \#_{\mathrm{DZ}}$ Feedback

$(\mathrm{BeDiaCo}$ : a_f8f9, 5,6-31 s)

In (4b) beginnt der Dialogzug von B in Form einer Antwort, wird mehrmals von A mit Feedback-Dialogzügen komplementiert und endet schließlich in (4f). Innerhalb ihrer Antwort produziert B vier IPn. Im Anschluss gibt A Feedback (4g), bevor sie in (4h) einen neuen Dialogzug und eine neue IP initiiert. B gibt in (4i) kurz Feedback, dann setzt A ihren Dialogzug in einer weiteren IP fort (4j). Ihr initiierender Dialogzug ist in diesem Beispiel nach (41) beendet (in realiter geht der DZ noch weiter). 
Das äh in Beispiel (4b) steht am Anfang eines beantwortenden Dialogzuges, nämlich einer W-Frage. Zugleich steht es auch zu Beginn einer IP. In seinem linksadjazenten Kontext steht je nach Betrachtung entweder das Wort Mensa von Versuchsperson A oder eine Pause in der Dialogspur von Versuchsperson B, in seinem rechtsadjazenten Kontext steht das Wort $i c h$. Nur aus der Transkription heraus lässt sich die phonetische Realisierung dieses äh nicht beschreiben, womit nach jetzigem Stand der Forschung ein Zusammenhang zwischen seiner Realisierungsform und seiner Position im Dialog weder angenommen noch ausgeschlossen werden kann. Eine solche Analyse ist jedoch - wie ich in diesem Kapitel argumentiere - geboten, um die auftretenden Muster in spontaner gesprochener Sprache linguistisch adäquat beschreiben und daraus kommunikative Gebrauchsmuster ableiten zu können. Mit der Form-Kontext-Hypothese (vgl. Abschnitt 3.2.1) postuliere ich, dass die dialogische Struktur mit der Form von Füllpartikeln, i. e. ihrer phonetischen Realisierung, interagiert.

Beispielsweise kann das äh in Beispiel (4b) initiale glottale Plosive zeigen und sich in seiner Vokalqualität, Dauer, Grundfrequenz und Phonationsart von anderen Füllpartikeln der gleichen Sprecherin unterscheiden (vgl. Abschnitt 2.2). In seiner spezifischen Position zwischen einer Pause und einem Laut in Beispiel (4b) ist die vokalische Form äh nach der Distributionsanalyse von Jessen (2012) in etwa $18 \%$ aller $\ddot{a} h$-Vorkommen vorgesehen (vgl. Abschnitt 3.2.2.1). Nach der Distributionsanalyse von (Leeuw 2007) steht eine $\ddot{a} h$-Form in nur $6 \%$ aller Pause-FP-Wort-Kontexte (vgl. Abschnitt 3.2.2.2). In seinem hierarchischen Kontext (zu Beginn einer Intonationsphrase) könnte es eine eher erhöhte $\mathrm{f}_{0}$ zeigen als an anderen Positionen (vgl. Abschnitt 3.2.3.2).

Über die Einbindung von Dialogzügen als hierarchisch übergeordnete und damit potenziell verstärkend wirkende prosodisch-linguistische Grenzen ist nichts bekannt, es ist daher durchaus vorstellbar, dass auch der Dialogzug (Antwort auf eine WFrage) als höhergeordneter Kontext eine verstärkende Wirkung auf die phonetische Realisierung (in diesem Fall die Dauer) von Segmenten an dieser Position ausübt.

Zusammenfassend ist über die phonetische Realisierung der Füllpartikeln im Zusammenhang von Dialogzügen - zumal im Deutschen - kaum etwas bekannt. Bisherige Studien haben vor allem aufgabenbasierte Dialoge untersucht und geben keine nähere Beschreibung der phonetischen Realisierung in bestimmten Positionen des 
Dialogzugs. Aus den zusammengefassten Ergebnissen bisheriger Studien über die phonetischen Realisierungen in anderen hierarchischen Kontexten wie syntaktischen Phrasen und Intonationsphrasen sowie in sequenziellen Kontexten wie Pausen, Lauten und Wörtern lässt sich die Hypothese aufstellen, dass auch Dialogzüge bestimmte systematisch vorkommende Ausprägungen phonetischer Realisierungen zeigen, da beispielsweise der Beginn einer Intonationsphrase mit dem Beginn eines Dialogzuges zusammenfallen kann und somit analog zu anderen additiven prosodischen Stärkungsprozessen (vgl. initiale Stärkung, finale Dehnung) ausgeprägtere phonetische Muster erwartbar sind. Die Beschreibung dieser Muster für solche hierarchisch höhergeordnete Chunks in einem Dialog, nämlich Dialogzüge, ist bisher nur rudimentär für die Beschreibung der Distribution von Füllpartikeln erfolgt - ihre phonetische Form wurde nicht beschrieben. Die weitere Beforschung dieses Zusammenhangs ist jedoch notwendig, um die hohe Variabilität der phonetischen Füllpartikelformen (vgl. Kapitel 2) einordnen zu können.

\subsection{Paralinguistische Variation}

Als paralinguistische Variation bezeichne ich diejenigen Freiheitsgrade in der Realisierung von Füllpartikeln, die nicht von bestimmten linguistischen Auftretenspositionen und -kontexten abhängig sind (vgl. Abschnitt 3.2), sondern sprecher- und situationsspezifisch erklärt werden können. Trotz sprecherspezifischer Unterschiede kann die Beantwortung der Forschungsfrage durch die Wahl geeigneter statistischer Methoden dennoch gewährleistet werden. Bei situationsspezifischen Unterschieden gilt es jedoch, die Aufnahmesituation a priori zu kontrollieren.

\subsubsection{Sprecherspezifität}

Sprecher*innen zeigen eine große Formvariabilität in der Produktion von Füllpartikeln in bestimmten Positionen sowie große Frequenzunterschiede. So finden Clark \& Fox Tree (2002: 97) eine Spanne von 1,2-88,5 Füllpartikeln je 1000 Wörter für die 65 untersuchten Versuchspersonen (Median 17,3). Andere Studien berichten über idiosynkratische Präferenzen bei der Wahl und Positionierung von V- und VN-Formen (Leeuw 2007: 109; Shriberg 1994: 156).

Auch Alters- und Geschlechterunterschiede scheinen sich sowohl auf die Frequenz 
als auch auf die Präferenz für bestimmte Füllpartikelformen auszuwirken. Männliche Englischsprecher zeigen eine signifikant höhere Füllpartikelrate pro Wort als Frauen (ca. $2,7 \%$ vs. $1,4 \%$ bei Shriberg 1994: 148, Abb. 29, 3,04\% vs. 2,07\% bei Bortfeld et al. 2001: 139). In aufgabenbasierten Dialogen drei verschiedener Altersgruppen (im Mittel 28, 47 und 67 Jahre alt) produziert die ältere Gruppe signifikant häufiger Disfluencies $^{80}$ als die mittlere und jüngere Gruppe, während die beiden letzteren sich nicht unterschieden (ebd.). Alterseffekte können in dieser Arbeit nicht evaluiert werden, da alle Versuchspersonen aus einer ähnlichen Kohorte stammen (20-31 Jahre).

Die männlichen Probanden in den deutschsprachigen Interviews von Jessen (2012: $152)$ verwenden die V-Form insgesamt häufiger als die VN-Form (2,48 V/min vs. 1,18 $\mathrm{VN} / \mathrm{min}$ ), jedoch verwenden einige Sprecher fast nur die V-Form, andere fast nur die VN-Form. Auch im Englischen wird in Interviews beobachtet, dass Männer mehr V-als VN-Formen produzieren (McDougall \& Duckworth 2017: 22). Passend dazu beobachten Wieling et al. (2016), dass Frauen und jüngere Menschen jeweils ein größeres VN-zu-V-Verhältnis aufweisen als Männer und ältere Menschen (vgl. auch Tottie 2013). Im Amerikanischen Englisch liegt das VN-zu-V-Verhältnis im Speed Dating Corpus (Jurafsky et al. 2009), je nach Transkriptionsquelle, für Frauen bei 1,8-2,4, für Männer bei 0,5-0,7 (Acton 2011: 4). Andererseits finden Laserna et al. (2014) in einer Studie alltäglicher Konversationen englischer Muttersprachlerinnen und Muttersprachler außerhalb des Labors mit 263 Männern und Frauen keinen Geschlechtereffekt beim Gebrauch der V-/VN-Formen und nur einen schwachen Alterseffekt $(r=-0,12, p=0,05)$ beim Vergleich von vier Probandengruppen (17-19, 20-22, 23-34 und 35-69 Jahre) (ebd.: 333).

Akustisch gibt es eine starke Korrelation $(r=0,86)$ zwischen dem $\mathrm{F}_{1}$-Mittelwert von $u h$ und dem $\mathrm{F}_{1}$-Mittelwert von $u h m$ sowie zwischen dem $\mathrm{F}_{2}$-Mittelwert von $u h$ und dem $\mathrm{F}_{2}$-Mittelwert von $u \mathrm{hm}$ der einzelnen Sprecher in fiktiven Polizeibefragungen mit 60 männlichen englischsprachigen Sprechern (Hughes et al. 2016: 116). Dies deutet darauf hin, dass sich die Qualität des in der Mitte gemessenen Füllpartikelvokals in V-Formen innerhalb eines Sprechers nicht sonderlich von VN-Formen unterscheidet (vgl. ebd.), für die Formanttrajektorien ist das Bild jedoch eher divers (ebd.: 124). In dieser Arbeit wird sich herausstellen, dass der Vokal in V-Formen im

\footnotetext{
${ }^{80}$ Hierunter zählen in ihrer Studie auch Wiederholungen und Neustarts (Bortfeld et al. 2001: 134).
} 
Deutschen systematisch tiefer und anteriorer als der Vokal in VN-Formen produziert wird.

Keine der besprochenen Studien berücksichtigt direkte akustische oder weitere dialogstrukturelle Kontexte, um die etwaige Abhängigkeit der Distributionen von anderen Variablen zu überprüfen (zu diesem Kritikpunkt vgl. auch Hughes et al. 2016: 102 f.). Es ist beispielsweise nicht ausgeschlossen, dass sich die Dialogstrukturen oder -strategien der Sprecherinnen und Sprecher voneinander und untereinander unterscheiden. Die Herausforderung liegt also darin, über die sprecherspezifischen Frequenzen, Distributionen und Formen zu generalisieren, um systematische Faktoren ausfindig zu machen.

\subsubsection{Situationspezifität}

Die produzierte Füllpartikelanzahl kann von der Situation abhängen, in der sich ein*e Sprecher*in befindet. Der Einfluss von Alkohol reduziert die Füllpartikelanzahl (Christenfeld \& Creager 1996). Auch eine von außen festgelegte Sprechgeschwindigkeit reduziert Füllpartikeln (Christenfeld 1996: 4). Universitätsvorlesungen und Map-Task-Dialoge wirken sich im Portugiesischen nicht auf die Häufigkeit von Füllpartikeln aus (Moniz et al. 2014). Im Englischen steigt die Füllpartikelanzahl, wenn man mit einem Menschen statt zu einem Computer spricht (E. J. Walker et al. 2014: 265), im Schwedischen sinkt sie (Eklund 2004).

Manchmal reicht die bloße Anwesenheit anderer Personen, um die Disfluencyrate zu erhöhen (Broen \& Siegel 1972: 224). Schnadt \& Corley (2006) hingegen finden keinen Effekt einer zweiten Person im Raum. Das von Bell (1984) beschriebene $a u$ dience design besagt, dass sich Sprecherinnen und Sprecher bei der Wahl ihres Stils oder Registers nicht nur am Gegenüber, sondern zu einem gewissen Grad auch an anderen Personen (Dritten) orientieren, die nicht direkt am Gespräch teilnehmen, die aber bekannt und ratifiziert sind (auditors) oder unbekannt sind und zufällig das Gespräch mithören könnten (overhearers) (ebd.: 159). Dieser Effekt kann in der vorliegenden Arbeit nicht ganz ausgeschlossen werden, allerdings war der Experimentator nicht direkt mit in der Aufnahmekabine und auch während des Dialogs zumindest in $\mathrm{BeDiaCo}_{\mathrm{c}}$ nicht zu sehen. 


\subsection{Fazit und Forschungsdesiderat}

In diesem Kapitel habe ich die (multi-)funktionalen Aspekte von Füllpartikeln, ihre möglichen Auftretenskontexte und einige schon bekannte damit verbundene phonetische Realisierungen vorgestellt. Diese Arbeit wird die phonetische Realisierung von Füllpartikeln in ihren Auftretenskontexten eingehender analysieren (Form-KontextHypothese) und somit prüfen, ob die Formvariabilität durch ihre Kontexte erklärt werden kann. Verschiedene Vorarbeiten deuten darauf hin, dass dies eine fruchtbare Hypothese ist. Aufgrund der hohen Variabilität der Realisierungen und Kontexte ist ein detailliertes methodisches Vorgehen notwendig. Die Hypothese, dass die akustische Realisierung von ihrem Auftretenskontext abhängig ist, macht die Betrachtung sequenzieller, füllpartikeladjazenter Laute und Wörter nötig, um Auswirkungen des segmentalen Kontextes auf die Lautrealisierung auszuschließen. Somit orientiere ich mich an einem der von Local \& G. Walker (2005) aufgestellten Imperative für die methodologische Betrachtung phonetischer Strukturen in spontaner Sprache.

Treat all details at all levels as of potential relevance to the participants. [Kursivierung lt. Quelle-M. B.] We simply do not know, from the outset, which details might be of relevance to the participants and might have a communicative function. (ebd.: 122)

Neben der epistemischen Frage nach den ,Details‘, die jeder Fachbereich, der sich mit gesprochener Sprache beschäftigt, anders beantworten wird, kann trotz der sorgfältigen Beschreibung und Analyse der phonetischen Korrelate in dieser Arbeit keineswegs davon ausgegangen werden, dass andere als die hier betrachteten phonetischen Korrelate relevant für die Verbindung zwischen Form und Kontext sind oder dass andere als die untersuchten Faktoren einen Einfluss auf die phonetischen Korrelate von Füllpartikeln haben. Gerade der Detailreichtum der beeinflussenden Variable Kontext muss notwendigerweise - linguistisch begründet - reduziert werden, um zu einer abstrahierenden Aussage über den Zusammenhang von Kontext und Form zu gelangen.

Die Auswahl der potenziell beeinflussenden Kontexte auf die phonetische Form von Füllpartikeln priorisiere ich unter Betrachtung der bisherigen Forschung und unter den zeitlichen Restriktionen dieser Arbeit daher wie folgt. Als sequenzielle Kontexte werden die füllpartikeladjazenten Laute und Wörter analysiert (Mikrokontext). Hierunter fallen auch Pausen und Atmungsgeräusche. Für diese Kontexte 
existieren (zumindest fürs Englische) zahlreiche Studien, die einen Zusammenhang zwischen Kontext und Form nahelegen (vgl. Abschnitt 3.2.2). Als hierarchischer Kontext wird in den beiden folgenden Korpusstudien die Dialogstruktur betrachtet (Makrokontext, da diese Struktur höhergeordneter zusammengehörender Einheiten jedem spontanen Dialog innewohnt und in der Literatur erste Hinweise auf einen erklärenden Aspekt dieser Struktur vorhanden sind, die bisher nicht vertieft wurden (vgl. Abschnitt 3.2.3.3). Nach der explorativen Studie (vgl. Abschnitt 5.1.1) ziehe ich in der konfirmatorischen Studie zusätzlich Intonationsphrasen (vgl. Abschnitt 3.2.3.2) als hierarchischen Kontext hinzu, quasi als Mesokontext zwischen Mikro- und Makrokontexten, um eine weitere Verfeinerung der dialogstrukturellen Kontexte zu erreichen (vgl. hierzu auch Kapitel 5).

Während für die Überprüfung der in Abschnitt 2.3 aufgestellten Hypothese der Formvariabilität eine kontextunabhängige phonetische Analyse von Füllpartikelinstanzen ausreicht (was die Schwierigkeit nicht mindert), kann die Form-Kontext-Hypothese nur mithilfe linguistischer Kontexte überprüft werden. Hierbei sind klar unterscheidbare Kontexte vonnöten, um die vorkommenden Formen distributiv fassen zu können. Mithilfe des hier verwendeten kleinteiligen und unabhängig aufeinander aufbauenden Ansatzes der Mehrebenenannotation dieser Kontexte in gesprochenen Korpora spontansprachlicher aufgabenfreier Dialoge kann jeder Kontext sowohl für sich selbst analysiert als auch in den Zusammenhang anderer Kontexte gestellt werden. Die hier von mir aufgestellten Hypothesen, die Form-Kontext-Hypothese (vgl. Abschnitt 3.2.1) und die Hypothese der Formvariabilität (vgl. Abschnitt 2.3), emergieren aus dem aktuellen Forschungsstand heraus. Ihre Überprüfung ist daher ein notwendiger und fruchtbarer Schritt hin zu einem besseren Verständnis von Füllpartikeln. Die im Folgenden verwendete Methode der oberflächenbasierten Vergabe von Annotationswerten ermöglicht eine transparente, nachvollziehbare und gebrauchsbasierte Analyse der phonetischen Füllpartikelform in ihrem Kontext, da hier keine weitgreifenden funktionalen Interpretationen gezogen werden. Im Ergebnis der folgenden Studien ist es so möglich festzustellen, ob systematische Form-Kontext-Paare angenommen werden können. Erst im Anschluss enstünde somit eine solide und gebrauchsbasiert emergierte Wissensbasis, um diesen Form-Kontext-Paaren (und dies wäre ein offenes Desiderat für weitere Forschung außerhalb dieser Arbeit) kommunikative Funktionen zuzuschreiben. 
Open Access Dieses Kapitel wird unter der Creative Commons Namensnennung 4.0 International Lizenz (http://creativecommons.org/licenses/by/4.0/deed.de) veröffentlicht, welche die Nutzung, Vervielfältigung, Bearbeitung, Verbreitung und Wiedergabe in jeglichem Medium und Format erlaubt, sofern Sie den/die ursprünglichen Autor(en) und die Quelle ordnungsgemäß nennen, einen Link zur Creative Commons Lizenz beifügen und angeben, ob Änderungen vorgenommen wurden.

Die in diesem Kapitel enthaltenen Bilder und sonstiges Drittmaterial unterliegen ebenfalls der genannten Creative Commons Lizenz, sofern sich aus der Abbildungslegende nichts anderes ergibt. Sofern das betreffende Material nicht unter der genannten Creative Commons Lizenz steht und die betreffende Handlung nicht nach gesetzlichen Vorschriften erlaubt ist, ist für die oben aufgeführten Weiterverwendungen des Materials die Einwilligung des jeweiligen Rechteinhabers einzuholen.

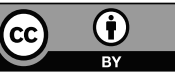

